MATHEMATICS OF COMPUTATION

Volume 67, Number 224, October 1998, Pages 1401-1422

S 0025-5718(98)00980-6

\title{
ACCELERATED SPECTRAL APPROXIMATION
}

\author{
RAFIKUL ALAM, REKHA P. KULKARNI, AND BALMOHAN V. LIMAYE
}

\begin{abstract}
A systematic development of higher order spectral analysis, introduced by Dellwo and Friedman, is undertaken in the framework of an appropriate product space. Accelerated analogues of Osborn's results about spectral approximation are presented. Numerical examples are given by considering an integral operator.
\end{abstract}

\section{INTRODUCTION}

In [10] Osborn considered numerical solution of an eigenvalue problem for a compact operator $T$ on a complex Banach space $X$ and obtained error estimates for the approximation of eigenvalues, eigenvectors and spectral subspaces, when a sequence $\left(T_{n}\right)$ of compact operators approximates $T$ in a collectively compact manner. In [11] Vainikko obtained similar results under (discrete) regular approximation. Subsequently, numerical solutions of eigenvalue problems for compact as well as noncompact operators have been studied extensively ([1], [3], [4], [6], [7], $[9])$.

In [5] Dellwo and Friedman developed a new approach to the spectral approximation of a compact operator by solving a polynomial eigenvalue problem of a higher degree. The eigenvalue problem associated with the $q$ th degree operator polynomial was referred to as the $q$ th order spectral analysis of $T, q=1,2, \ldots$ They proved that, if $\lambda$ is a nonzero eigenvalue of $T$ of algebraic multiplicity $m$ and ascent $l$, then the $q$ th order spectral analysis provides sets $\sigma_{q, n}$ of approximate spectra associated with $\lambda$, which satisfy the order relationship

$$
\max _{\mu \in \sigma_{q, n}}|\lambda-\mu|^{l}=O\left(\frac{1}{|\lambda|^{q}}\left\|\left(T-T_{n}\right)^{q} T_{n}\right\|\right) .
$$

Several numerical examples were considered to illustrate the effectiveness of higher order spectral analysis. However, the exact nature of the set $\sigma_{q, n}$ was not specified.

In this paper an attempt is made to develop a methodology for a systematic study of higher order spectral analysis. We transform a polynomial eigenvalue problem associated with a higher order spectral analysis to an equivalent ordinary eigenvalue problem in an appropriate product space. We thus obtain error estimates for accelerated approximation of eigenvalues, eigenvectors and spectral subspaces in exactly the same fashion as the ordinary spectral approximation. We consider a cluster $\Lambda$ of nonzero eigenvalues of $T$ of total algebraic multiplicity $m<\infty$ and show

Received by the editor September 11, 1995 and, in revised form, October 30, 1996.

1991 Mathematics Subject Classification. Primary 47A10, 47A58, 47A75, 65B99, 65J99.

Key words and phrases. Spectral approximation, higher order spectral analysis, eigenvalue of finite algebraic multiplicity, spectral projection, spectral subspace, eigenvector. 
that the $q$ th order spectral analysis provides exactly $m$ eigenvalues $\lambda_{q, n, 1}, \ldots, \lambda_{q, n, m}$ (counted according to their algebraic multiplicities) near the cluster $\Lambda$. If $\hat{\lambda}$ and $\hat{\lambda}_{q, n}$ denote the weighted averages of the eigenvalues in $\Lambda$ and of their approximations $\lambda_{q, n, 1}, \ldots, \lambda_{q, n, m}$, respectively, and if $\epsilon<\min \{|\lambda|: \lambda \in \Lambda\}$, then

$$
\left|\hat{\lambda}-\hat{\lambda}_{q, n}\right| \leq \frac{C}{\epsilon^{q-1}}\left\|\left(T-T_{n}\right)^{q} \mid R(P)\right\|,
$$

where $C$ is a constant independent of $n$ and $q$. This gives an accelerated analogue of Osborn's result for the approximation of the arithmetic mean $\hat{\lambda}$. We also prove that

$$
\left|\hat{\lambda}-\hat{\lambda}_{q, n}\right|=O\left(\frac{1}{\epsilon^{q-1}}\left\|\left(T-T_{n}\right)^{q} T_{n}\right\|\right) .
$$

This estimate improves upon the result of Dellwo and Friedman quoted earlier. If $\Lambda$ consists of a single eigenvalue $\lambda$ of ascent $l>1$, then error estimates for the approximation of $\lambda$ by individual eigenvalues $\lambda_{q, n, 1}, \ldots, \lambda_{q, n, m}$ is obtained by taking the $l$ th root of the above-mentioned error estimates. This slower convergence is illustrated in the last section by considering an integral operator. We give similar estimates for the approximation of eigenvectors and spectral subspaces as well. Results analogous to the improved error estimates given in Theorems 3 and 4 of [10] will be given in another paper. The methodology developed in this paper can be used to obtain accelerated analogues of various spectral refinement schemes which will be discussed in subsequent papers.

In Section 2, we give improved versions of results from [10] for the sake of completeness and for use in the subsequent sections. In Section 3, we develop a framework for higher order spectral analysis and obtain accelerated analogues of the results in [10] for the approximation of a cluster of eigenvalues, eigenvectors and spectral subspaces of a bounded linear operator.

\section{Preliminaries}

Throughout this paper $X$ will denote a complex Banach space and $B L(X)$ the Banach space of all bounded linear operators on $X$ along with the operator norm. For $T$ in $B L(X)$, let $\sigma(T)$ and $\rho(T)$ denote the spectrum and the resolvent set of $T$, respectively. We consider a nonempty subset $\Lambda$ of $\sigma(T) \backslash\{0\}$ which is separated from the rest of the spectrum of $T$ and from 0 by a simple closed positively oriented rectifiable curve $\Gamma$ lying in $\rho(T)$. Let $\ell(\Gamma)$ denote the length of $\Gamma$. For $z \in \rho(T)$, we let

so that

$$
R(z)=(T-z I)^{-1},
$$

$$
P=-\frac{1}{2 \pi i} \int_{\Gamma} R(z) d z
$$

is the spectral projection associated with $T$ and $\Lambda$. We assume that rank $P=$ $m<\infty$. Then $\Lambda$ consists of eigenvalues $\lambda_{1}, \ldots, \lambda_{m}$ of $T$, counted according to their algebraic multiplicities. For nonzero subspaces $Y$ and $Z$ of $X$, let

$$
\delta(Y, Z)=\sup \{\operatorname{dist}(y, Z): y \in Y,\|y\|=1\} .
$$

Then

$$
\hat{\delta}(Y, Z)=\max \{\delta(Y, Z), \delta(Z, Y)\}
$$

is known as the gap between $Y$ and $Z$. For $T \in B L(X)$, we denote by $R(T)$ and $N(T)$ the range space and the null space of $T$, respectively. 
In this section we consider a sequence $\left(T_{n}\right)$ in $B L(X)$ which satisfies

(H1) : $\left(\left\|T_{n}\right\|\right)$ is a bounded sequence,

(H2) : $\left\|\left(T-T_{n}\right) T\right\| \rightarrow 0$ and $\left\|\left(T-T_{n}\right) T_{n}\right\| \rightarrow 0$ as $n \rightarrow \infty$.

While it may be possible to weaken these hypotheses to some extent, they allow a simplicity in the presentation and are easy to check in several important examples. For $z \in \rho\left(T_{n}\right)$, we let

$$
R_{n}(z)=\left(T_{n}-z I\right)^{-1}
$$

Lemma 2.1 (Nair [9]). Let $E$ be a closed subset of $\rho(T) \backslash\{0\}$ and $\delta=$ $\min \{|z|: z \in E\}$. Then there is a constant $c_{1}$ such that

$$
\max _{z \in E}\|R(z)\| \leq c_{1} .
$$

Let $n_{0}$ be a positive integer such that

$$
\left\|\left(T-T_{n}\right)^{2}\right\|<\delta^{2} \text { and } c_{1}\left\|\left(T-T_{n}\right) T_{n}\right\| \leq \frac{\delta}{2}
$$

for all $n \geq n_{0}$. Then $E \subset \rho\left(T_{n}\right)$ and

$$
\max _{z \in E}\left\|R_{n}(z)\right\| \leq 2 c_{1}\left[1+\frac{\left\|T-T_{n}\right\|}{\delta}\right] \leq c_{2}
$$

for some constant $c_{2}$ and all $n \geq n_{0}$.

For a proof of this result we refer to [9]. Letting $E=\Gamma$ in Lemma 2.1, we see that $\Gamma \subset \rho\left(T_{n}\right)$ for all large $n$. Let

$$
P_{n}=-\frac{1}{2 \pi i} \int_{\Gamma} R_{n}(z) d z
$$

denote the spectral projection associated with $T_{n}$ and $\Gamma$. It can be seen, as in the proof of Theorem 3.1 of [9], that

$$
\left\|\left(P-P_{n}\right) P\right\| \rightarrow 0 \text { and }\left\|\left(P-P_{n}\right) P_{n}\right\| \rightarrow 0 \text { as } n \rightarrow \infty .
$$

Hence rank $P_{n}=\operatorname{rank} P=m$ for all large $n$ (cf. [9], Proposition 2.2.).

Theorem 2.2 (Osborn [10]). For all large n,

$$
\hat{\delta}\left(R(P), R\left(P_{n}\right)\right) \leq \frac{\ell(\Gamma)}{\pi} c_{1} c_{2} \min \left\{\left\|\left(T-T_{n}\right)_{\mid R(P)}\right\|,\left\|\left(T-T_{n}\right)_{\mid R\left(P_{n}\right)}\right\|\right\},
$$

where $c_{1}$ and $c_{2}$ are as in Lemma 2.1 with $E=\Gamma$.

Proof. The proof of Theorem 1 of [10] shows that

$$
\delta\left(R(P), R\left(P_{n}\right)\right) \leq \frac{\ell(\Gamma)}{2 \pi} c_{1} c_{2}\left\|\left(T-T_{n}\right)_{\mid R(P)}\right\| .
$$

Since, with $\delta=\min \{|z| \in \Gamma\}$,

$$
\left\|\left(T-T_{n}\right)_{\mid R(P)}\right\| \leq\left\|\left(T-T_{n}\right) P\right\| \leq \frac{\ell(\Gamma)}{2 \pi \delta} c_{1}\left\|\left(T-T_{n}\right) T\right\| \rightarrow 0,
$$

as $n \rightarrow \infty$, we take $n_{0}$ so large that $\delta\left(R(P), R\left(P_{n}\right)\right) \leq 1 / 2$.

As $\operatorname{dim} R\left(P_{n}\right)=\operatorname{dim} R(P)<\infty$, by a result given by Kato [8],

$$
\delta\left(R\left(P_{n}\right), R(P)\right) \leq \frac{\delta\left(R(P), R\left(P_{n}\right)\right)}{1-\delta\left(R(P), R\left(P_{n}\right)\right)} \leq 2 \delta\left(R(P), R\left(P_{n}\right)\right) .
$$

Thus

$$
\hat{\delta}\left(R(P), R\left(P_{n}\right)\right) \leq \frac{\ell(\Gamma)}{\pi} c_{1} c_{2}\left\|\left(T-T_{n}\right)_{\mid R(P)}\right\| .
$$


By interchanging the roles of $T$ and $T_{n}$, we obtain

$$
\hat{\delta}\left(R\left(P_{n}\right), R(P)\right) \leq \frac{\ell(\Gamma)}{\pi} c_{1} c_{2}\left\|\left(T_{n}-T\right)_{\mid R\left(P_{n}\right)}\right\| .
$$

Since rank $P_{n}=m$ for all large $n, \sigma\left(T_{n}\right) \cap$ Int $\Gamma$ consists of $m$ eigenvalues $\lambda_{n, 1}, \ldots, \lambda_{n, m}$ of $T_{n}$, counted according to their algebraic multiplicities. Let

$$
\hat{\lambda}_{n}=\frac{\lambda_{n, 1}+\cdots+\lambda_{n, m}}{m}
$$

denote their arithmetic mean.

Theorem 2.3 (Osborn [10]). For all large n, the maps

$$
A_{n}=P_{n \mid R(P)}: R(P) \rightarrow R\left(P_{n}\right) \text { and } B_{n}=P_{\mid R\left(P_{n}\right)}: R\left(P_{n}\right) \rightarrow R(P)
$$

are isomorphisms, $\left\|A_{n}^{-1}\right\| \leq 2,\left\|B_{n}^{-1}\right\| \leq 2$ and

$$
\left|\hat{\lambda}-\hat{\lambda}_{n}\right| \leq 2 \min \left\{\left\|P_{n}\right\|\left\|\left(T-T_{n}\right)_{\mid R(P)}\right\|,\|P\|\left\|\left(T-T_{n}\right)_{\mid R\left(P_{n}\right)}\right\|\right\} .
$$

If $\Lambda=\{\lambda\}$ and the ascent of $\lambda$ equals $l$, then for each $j=1, \ldots, m$,

$$
\left|\lambda-\lambda_{n, j}\right|^{l} \leq 2 \min \left\{c_{n}\left\|P_{n}\right\|\left\|\left(T-T_{n}\right)_{\mid R(P)}\right\|, d_{n}\|P\|\left\|\left(T-T_{n}\right)_{\mid R\left(P_{n}\right)}\right\|\right\},
$$

where

$$
\begin{aligned}
& c_{n}=\sum_{k=0}^{l-1}\left\|\lambda I_{\mid R(P)}-A_{n}^{-1} T_{n} A_{n}\right\|^{l-1-k}\left\|\lambda I_{\mid R(P)}-T_{\mid R(P)}\right\|^{k} \\
& d_{n}=\sum_{k=0}^{l-1}\left\|\lambda I_{\mid R\left(P_{n}\right)}-T_{n \mid R\left(P_{n}\right)}\right\|^{l-1-k}\left\|\lambda I_{\mid R\left(P_{n}\right)}-B_{n}^{-1} T B_{n}\right\|^{k} .
\end{aligned}
$$

Proof. The argument given in the proof of Theorem 2 of [10] shows that $A_{n}$ is bijective and $\left\|A_{n}^{-1}\right\| \leq 2$ for all large $n$. The same argument shows that $B_{n}$ is bijective and $\left\|B_{n}^{-1}\right\| \leq 2$ for all large $n$. Define $\hat{T}=T_{\mid R(P)}$ and $\hat{T}_{n}=A_{n}^{-1} T_{n} A_{n}$. Then

$$
\begin{aligned}
\left|\hat{\lambda}-\hat{\lambda}_{n}\right| & =\frac{1}{m}\left|\operatorname{trace}\left(\hat{T}-\hat{T}_{n}\right)\right| \leq\left\|\hat{T}-\hat{T}_{n}\right\| \\
& \left.=\sup _{\|}\left\|A_{n}^{-1} P_{n}\left(T-T_{n}\right) x\right\|: x \in R(P),\|x\|=1\right\} \\
& \leq 2\left\|P_{n}\right\|\left\|\left(T-T_{n}\right)_{\mid R(P)}\right\| .
\end{aligned}
$$

If $\Lambda=\{\lambda\}$ and the ascent of $\lambda$ is $l$, then since $\left(\lambda I_{\mid R(P)}-\hat{T}\right)^{l}=0$, we have for $j=1, \ldots, m$

$$
\begin{aligned}
\left|\lambda-\lambda_{n, j}\right|^{l} & \leq\left\|\left(\lambda I_{\mid R(P)}-\hat{T}_{n}\right)^{l}\right\|=\left\|\left(\lambda I_{\mid R(P)}-\hat{T}_{n}\right)^{l}-\left(\lambda I_{\mid R(P)}-\hat{T}\right)^{l}\right\| \\
& =\left\|\sum_{k=0}^{l-1}\left(\lambda I_{\mid R(P)}-\hat{T}_{n}\right)^{l-1-k}\left(\hat{T}-\hat{T}_{n}\right)\left(\lambda I_{\mid R(P)}-\hat{T}\right)^{k}\right\| \\
& \leq c_{n}\left\|\hat{T}-\hat{T}_{n}\right\| .
\end{aligned}
$$

Similarly, defining $\hat{T}_{n}=T_{n \mid R\left(P_{n}\right)}$ and $\hat{T}=B_{n}^{-1} T B_{n}$, we obtain the other estimates. The proof of the estimates for $\left|\lambda-\lambda_{n, j}\right|^{l}$ is adapted from [4], p. 685.

Let $\Lambda=\{\lambda\}$ and $l$ be the ascent of $\lambda$. We state the following theorem from [10]. 
Theorem 2.4 (Osborn [10]). Let $\lambda_{n}$ be an eigenvalue of $T_{n}$ such that $\lambda_{n} \rightarrow \lambda$ as $n \rightarrow \infty$. Suppose that $w_{n} \in N\left(T_{n}-\lambda_{n} I\right)$ with $\left\|w_{n}\right\|=1$. Then there is some $u_{n} \in N(T-\lambda I)$ such that

$$
\left\|u_{n}-w_{n}\right\| \leq c\left\{\left\|\left(T-T_{n}\right)_{\mid R(P)}\right\|\right\}^{1 / l},
$$

where $c$ is a constant independent of $n$.

\section{A FRAMEWORK FOR HIGHER ORDER APPROXIMATION}

Let $q$ be a positive integer and $\mathbf{X}_{q}$ denote the set of all column vectors $\mathbf{x}=$ $\left[x_{1}, \ldots, x_{q}\right]^{t}$ with $x_{1}, \ldots, x_{q}$ in $X$. Define

$$
\|\mathbf{x}\|_{\infty}=\max \left\{\left\|x_{j}\right\|: j=1, \ldots, q\right\} .
$$

Then $\mathbf{X}_{q}$ is a Banach space with respect to the norm \|\|$_{\infty}$. We shall identify the adjoint space of $\mathbf{X}_{q}$ with the set of all column vectors $\mathbf{x}^{*}=\left[x_{1}^{*}, \ldots, x_{q}^{*}\right]^{t}$ with $x_{1}^{*}, \ldots, x_{q}^{*}$ in $X^{*}$. Define

$$
\left\|\mathbf{x}^{*}\right\|_{1}=\left\|x_{1}^{*}\right\|+\cdots+\left\|x_{q}^{*}\right\|
$$

If we let

$$
\left\langle\mathbf{x}, \mathbf{x}^{*}\right\rangle=\left\langle x_{1}, x_{1}^{*}\right\rangle+\cdots+\left\langle x_{q}, x_{q}^{*}\right\rangle
$$

then it is clear that $\left|\left\langle\mathbf{x}, \mathbf{x}^{*}\right\rangle\right| \leq\|\mathbf{x}\|_{\infty}\left\|\mathbf{x}^{*}\right\|_{1}$. We have $\mathbf{X}_{1}=X$ and we let $\mathbf{T}_{1}=T$. Now let $q \geq 2$. Consider the operator $\mathbf{T}_{q}: \mathbf{X}_{q} \rightarrow \mathbf{X}_{q}$ given by

$$
\mathbf{T}_{q}\left[x_{1}, \ldots, x_{q}\right]^{t}=\left[T x_{1}, x_{1}, \ldots, x_{q-1}\right]^{t} .
$$

Then $\mathbf{T}_{q}$ can be written as the $q \times q$ matrix

$$
\left[\begin{array}{ccccc}
T & 0 & \cdots & \cdots & 0 \\
I & 0 & \cdots & \cdots & 0 \\
0 & \ddots & & & \vdots \\
\vdots & & \ddots & & \vdots \\
0 & \cdots & 0 & I & 0
\end{array}\right] .
$$

We have $\left\|\mathbf{T}_{q}\right\|_{\infty}=\max \{1,\|T\|\}$. For nonzero $z \in \mathbb{C}$, it can be easily seen that $\mathbf{T}_{q}-z \mathbf{I}_{q}$ is invertible if and only if $T-z I$ is invertible, and then $\left(\mathbf{T}_{q}-z \mathbf{I}_{q}\right)^{-1}$ can be written as the $q \times q$ matrix

$$
\left[\begin{array}{cccc}
R(z) & 0 & \ldots & 0 \\
\frac{R(z)}{z} & -\frac{I}{z} & \ldots & 0 \\
\vdots & \vdots & \ddots & \vdots \\
\frac{R(z)}{z^{q-1}} & -\frac{I}{z^{q-1}} & \ldots & -\frac{I}{z}
\end{array}\right]
$$

Thus $\sigma\left(\mathbf{T}_{q}\right) \backslash\{0\}=\sigma(T) \backslash\{0\}$. In particular, $\Gamma \subset \rho\left(\mathbf{T}_{q}\right)$ and $\sigma\left(\mathbf{T}_{q}\right) \cap \operatorname{Int} \Gamma=\Lambda$. Let

$$
\mathbf{P}_{q}=-\frac{1}{2 \pi i} \int_{\Gamma}\left(\mathbf{T}_{q}-z \mathbf{I}_{q}\right)^{-1} d z
$$

denote the spectral projection associated with $\mathbf{T}_{q}$ and $\Lambda$. Since 0 lies outside $\Gamma$, we have

$$
\mathbf{P}_{q}\left[x_{1}, \ldots, x_{q}\right]^{t}=\left[P x_{1}, S_{1} x_{1}, \ldots, S_{q-1} x_{1}\right]^{t},
$$


where

$$
S_{j}=-\frac{1}{2 \pi i} \int_{\Gamma} \frac{R(z)}{z^{j}} d z, \quad j=1, \ldots, q-1 .
$$

Now $\left[x_{1}, \ldots, x_{q}\right]^{t} \in R\left(\mathbf{P}_{q}\right)$ if and only if $x_{1} \in R(P), x_{2}=S_{1} x_{1}, \ldots, x_{q}=S_{q-1} x_{1}$. Hence the operator $J_{q}: R(P) \rightarrow R\left(\mathbf{P}_{q}\right)$ given by

$$
J_{q} x=\left[x, S_{1} x, \ldots, S_{q-1} x\right]^{t}, \quad x \in R(P),
$$

is a surjective isomorphism and

$$
\text { rank } \mathbf{P}_{q}=\operatorname{rank} P=m \text {. }
$$

Next, the spectral projection associated with $\mathbf{T}_{q}^{*}: \mathbf{X}_{q}^{*} \rightarrow \mathbf{X}_{q}^{*}$ and $\bar{\Lambda}=\{\bar{\lambda}: \lambda \in \Lambda\}$ is given by

$$
\mathbf{P}_{q}^{*}\left[x_{1}^{*}, \ldots, x_{q}^{*}\right]^{t}=\left[P^{*} x_{1}^{*}+S_{1}^{*} x_{2}^{*}+\cdots+S_{q-1}^{*} x_{q}^{*}, 0, \ldots, 0\right]^{t} .
$$

Thus $\left[x_{1}^{*}, \ldots, x_{q}^{*}\right]^{t} \in R\left(\mathbf{P}_{q}^{*}\right)$ if and only if $x_{1}^{*} \in R\left(P^{*}\right), x_{2}^{*}=\cdots=x_{q}^{*}=0$. Hence the operator $K_{q}: R\left(P^{*}\right) \rightarrow R\left(\mathbf{P}_{q}^{*}\right)$ given by

$$
K_{q} x^{*}=\left[x^{*}, 0, \ldots, 0\right]^{t}, \quad x^{*} \in R\left(P^{*}\right),
$$

is a surjective isomorphism. Also,

$$
\left\langle J_{q} x, K_{q} x^{*}\right\rangle=\left\langle x, x^{*}\right\rangle \quad \text { for all } x \in R(P) \text { and } x^{*} \in R\left(P^{*}\right) .
$$

Next, consider $\lambda \in \Lambda$ and let $P_{\lambda}$ (resp. $\mathbf{P}_{\lambda}$ ) denote the spectral projection associated with $T$ and $\lambda$ (resp., $\mathbf{T}_{q}$ and $\lambda$ ). Then rank $\mathbf{P}_{\lambda}=\operatorname{rank} P_{\lambda}$ just as before, so that the algebraic multiplicity of $\lambda$ as an eigenvalue of $\mathbf{T}_{q}$ is the same as the algebraic multiplicity of $\lambda$ as an eigenvalue of $T$. Consider

$$
D_{\lambda}=P_{\lambda}(T-\lambda I) \quad \text { and } \quad \mathbf{D}_{\lambda}=\mathbf{P}_{\lambda}\left(\mathbf{T}_{q}-\lambda \mathbf{I}_{q}\right) .
$$

Then

$$
\begin{aligned}
\mathbf{D}_{\lambda}\left[x_{1}, \ldots, x_{q}\right]^{t} & =\mathbf{P}_{\lambda}\left[(T-\lambda I) x_{1}, x_{1}-\lambda x_{2}, \ldots, x_{q-1}-\lambda x_{q}\right]^{t} \\
& =\left[P_{\lambda}(T-\lambda I) x_{1}, S_{\lambda, 1}(T-\lambda I) x_{1}, \ldots, S_{\lambda, q-1}(T-\lambda I) x_{1}\right]^{t},
\end{aligned}
$$

where

$$
S_{\lambda, j}=-\frac{1}{2 \pi i} \int_{\Gamma_{\lambda}} \frac{R(z)}{z^{j}} d z, \quad j=1, \ldots, q-1,
$$

$\Gamma_{\lambda}$ being a simple closed curve which isolates $\lambda$ from the rest of $\sigma(T)$ and from 0 . By the usual techniques of contour integration, it can be seen that $S_{\lambda, j} P_{\lambda}=S_{\lambda, j}=$ $P_{\lambda} S_{\lambda, j}$ for $j=1, \ldots, q-1$. Hence

$$
\mathbf{D}_{\lambda}\left[x_{1}, \ldots, x_{q}\right]^{t}=\left[D_{\lambda} x_{1}, S_{\lambda, 1} D_{\lambda} x_{1}, \ldots, S_{\lambda, q-1} D_{\lambda} x_{1}\right]^{t} .
$$

Similarly, for $k=2,3, \ldots$, we have

$$
\mathbf{D}_{\lambda}^{k}\left[x_{1}, \ldots, x_{q}\right]^{t}=\left[D_{\lambda}^{k} x_{1}, S_{\lambda, 1} D_{\lambda}^{k} x_{1}, \ldots, S_{\lambda, q-1} D_{\lambda}^{k} x_{1}\right]^{t} .
$$

Thus for any positive integer $k$, we have $\mathbf{D}_{\lambda}^{k}=0$ if and only if $D_{\lambda}^{k}=0$. This shows that the ascent of $\lambda$ as an eigenvalue of $\mathbf{T}_{q}$ is the same as the ascent of $\lambda$ as an eigenvalue of $T$. Thus we see that the eigenvalue problem

$$
T \phi=\lambda \phi, \quad \phi \in X, \quad \phi \neq 0
$$

is equivalent to the eigenvalue problem

$$
\mathbf{T}_{q} \Phi_{q}=\lambda \Phi_{q}, \quad \Phi_{q} \in \mathbf{X}_{q}, \quad \Phi_{q} \neq 0
$$

for each $q=2,3, \ldots$ 
Let $\left(T_{n}\right)$ be a sequence in $B L(X)$. We have $\mathbf{X}_{1}=X$ and we let $\mathbf{T}_{1, n}=T_{n}$. Let now $q \geq 2$. For $n=1,2, \ldots$, let $\Delta_{n}=T-T_{n}$ and consider the operator $\mathbf{T}_{q, n}: \mathbf{X}_{q} \rightarrow \mathbf{X}_{q}$ given by

$$
\mathbf{T}_{q, n}\left[x_{1}, \ldots, x_{q}\right]^{t}=\left[\sum_{j=0}^{q-1} \Delta_{n}^{j} T_{n} x_{j+1}, x_{1}, \ldots, x_{q-1}\right]^{t} .
$$

Then $\mathbf{T}_{q, n}$ can be written as the $q \times q$ matrix

$$
\left[\begin{array}{ccccc}
T_{n} & \Delta_{n} T_{n} & \cdots & \cdots & \Delta_{n}^{q-1} T_{n} \\
I & 0 & \cdots & \cdots & 0 \\
0 & \ddots & & & \vdots \\
\vdots & & \ddots & & \vdots \\
0 & 0 & \cdots & I & 0
\end{array}\right] .
$$

We consider the eigenvalue problem

$$
\mathbf{T}_{q, n} \Phi_{q, n}=\lambda_{q, n} \Phi_{q, n}, \quad \Phi_{q, n} \in \mathbf{X}_{q}, \quad \Phi_{q, n} \neq 0 .
$$

Then it is easy to see that $\Phi_{q, n}=\left[\phi_{q, n}, \frac{\phi_{q, n}}{\lambda_{q, n}}, \ldots, \frac{\phi_{q, n}}{\left(\lambda_{q, n}\right)^{q}}\right]^{t}$, where the first component $\phi_{q, n} \in X$ satisfies

$$
\left(\left(\lambda_{q, n}\right)^{q} I-\sum_{j=0}^{q-1}\left(\lambda_{q, n}\right)^{q-1-j} \Delta_{n}^{j} T_{n}\right) \phi_{q, n}=0
$$

(cf. (2.4) of [5]). The case $q=1$ is considered in Section 2. For the rest of the paper we let $q \geq 2$ and assume that

(H1) $\left(\left\|T_{n}\right\|\right)$ is a bounded sequence,

(H2') $\left\|\left(T-T_{n}\right)^{2}\right\| \rightarrow 0$ as $n \rightarrow \infty$.

Note that the results of Section 2, where $q=1$, do not hold under the hypotheses (H1) and (H2'). As a simple example, consider $X=\mathbb{C}^{2}$ and

$$
T=\left[\begin{array}{cc}
a & a b \\
1 & b
\end{array}\right], \quad T_{n}=\left[\begin{array}{cc}
a_{n} & a_{n} b_{n} \\
0 & b_{n}
\end{array}\right], \quad n=1,2, \ldots,
$$

where $a, b, a_{n}, b_{n}$ are nonzero complex numbers with $b \neq-a$ and $a_{n} \rightarrow a, b_{n} \rightarrow b$ as $n \rightarrow \infty$. Then $\sigma(T)=\{0, a+b\}$, while $\sigma\left(T_{n}\right)=\left\{a_{n}, b_{n}\right\}$. Thus the nonzero simple eigenvalue $a+b$ of $T$ is not approximated by the nonzero eigenvalues $a_{n}$ and $b_{n}$ of $T_{n}$.

We have

$$
\begin{aligned}
\left\|\mathbf{T}_{q, n}\right\|_{\infty} & \leq \max \left\{1,\left\|T_{n}\right\|+\left\|\Delta_{n} T_{n}\right\|+\cdots+\left\|\Delta_{n}^{q-1} T_{n}\right\|\right\}, \\
\left\|\left(\mathbf{T}_{q}-\mathbf{T}_{q, n}\right) \mathbf{T}_{q}\right\|_{\infty} & \leq\left\|\Delta_{n}^{2}\right\|+\left\|\Delta_{n}^{2} T_{n}\right\|+\cdots+\left\|\Delta_{n}^{q-1} T_{n}\right\|, \\
\left\|\left(\mathbf{T}_{q}-\mathbf{T}_{q, n}\right) \mathbf{T}_{q, n}\right\|_{\infty} & =\left\|\Delta_{n}^{q} T_{n}\right\| .
\end{aligned}
$$

Since $\left\|\Delta_{n}^{2}\right\| \rightarrow 0$ as $n \rightarrow \infty$, there is an integer $n_{0}$ such that $\left\|\Delta_{n}^{2}\right\| \leq 1 / 2$ for all $n \geq n_{0}$. As

$$
\left\|\Delta_{n}^{j} T_{n}\right\| \leq \begin{cases}\left\|T_{n}\right\|\left\|\Delta_{n}^{2}\right\|^{j / 2}, & \text { if } j \text { is even } \\ \left\|\Delta_{n} T_{n}\right\|\left\|\Delta_{n}^{2}\right\|^{(j-1) / 2}, & \text { if } j \text { is odd }\end{cases}
$$


and $\sum_{k=0}^{\infty}\left\|\Delta_{n}^{2}\right\|^{k} \leq 2$ for $n \geq n_{0}$, we have

$$
\begin{aligned}
\left\|\mathbf{T}_{q, n}\right\|_{\infty} & \leq \max \left\{1,2\left(\left\|T_{n}\right\|+\left\|\Delta_{n} T_{n}\right\|\right)\right\}, \\
\left\|\left(\mathbf{T}_{q}-\mathbf{T}_{q, n}\right) \mathbf{T}_{q}\right\|_{\infty} & \leq 2\left(\left\|T_{n}\right\|+\left\|\Delta_{n}^{2} T_{n}\right\|\right)\left\|\Delta_{n}^{2}\right\|, \\
\left\|\left(\mathbf{T}_{q}-\mathbf{T}_{q, n}\right) \mathbf{T}_{q, n}\right\|_{\infty} & \leq \max \left\{\left\|T_{n}\right\|,\left\|\Delta_{n} T_{n}\right\|\right\}\left\|\Delta_{n}^{2}\right\| .
\end{aligned}
$$

As $\left\|T_{n}\right\|$ is bounded, this shows that $\left\|\mathbf{T}_{q, n}\right\|_{\infty}$ is bounded in $q$ and $n$,

$$
\left\|\left(\mathbf{T}_{q}-\mathbf{T}_{q, n}\right) \mathbf{T}_{q}\right\|_{\infty} \rightarrow 0 \quad \text { and } \quad\left\|\left(\mathbf{T}_{q}-\mathbf{T}_{q, n}\right) \mathbf{T}_{q, n}\right\|_{\infty} \rightarrow 0
$$

as $n \rightarrow \infty$, uniformly for $q=2,3, \ldots$. Thus the sequence $\left(\mathbf{T}_{q, n}\right)$ satisfies the hypotheses (H1) and (H2) of Section 2 uniformly in $q=2,3, \ldots$.

The following two identities will be useful. For nonzero $z$ in $\mathbb{C}$,

$$
\begin{gathered}
\frac{z I-\Delta_{n}}{z}\left(z I-\sum_{j=0}^{q-1} \frac{\Delta_{n}^{j} T_{n}}{z^{j}}\right)=z I-T+\frac{\Delta_{n}^{q} T_{n}}{z^{q}}, \\
z I-\sum_{j=0}^{q-1} \frac{\Delta_{n}^{j} T_{n}}{z^{j}}-\sum_{j=0}^{q} \frac{\Delta_{n}^{j}(z I-T)}{z^{j}}=\frac{\Delta_{n}^{q} T}{z^{q}} .
\end{gathered}
$$

3.1. Main results. We prove an important estimate.

Proposition 3.1. Let $\min \{|\lambda|: \lambda \in \Lambda\}>\epsilon$. If the curve $\Gamma$ lies in $\{z \in \mathbb{C}:|z| \geq \epsilon\}$ and $c_{1}=\max _{z \in \Gamma}\|R(z)\|$, then

$$
\left\|\left(\mathbf{T}_{q}-\mathbf{T}_{q, n}\right)_{\mid R\left(\mathbf{P}_{q}\right)}\right\|_{\infty} \leq \frac{\ell(\Gamma) c_{1}}{2 \pi \epsilon^{q-1}}\left\|\left(T-T_{n}\right)^{q} \mid R(P)\right\|
$$

for all $n$ and $q$.

Proof. For $\mathbf{x}=\left[x_{1}, \ldots, x_{q}\right]^{t} \in \mathbf{X}_{q}$, we have

$$
\left(\mathbf{T}_{q}-\mathbf{T}_{q, n}\right) \mathbf{x}=\left[\Delta_{n} x_{1}-\sum_{j=1}^{q-1} \Delta_{n}^{j} T_{n} x_{j+1}, 0, \ldots, 0\right]^{t}
$$

Since

$$
\mathbf{P}_{q} \mathbf{x}=\left[P x_{1}, S_{1} x_{1}, \ldots, S_{q-1} x_{1}\right]^{t},
$$

it follows that

$$
\left(\mathbf{T}_{q}-\mathbf{T}_{q, n}\right) \mathbf{P}_{q} \mathbf{x}=\left[\left(\Delta_{n} P-\sum_{j=1}^{q-1} \Delta_{n}^{j} T_{n} S_{j}\right) x_{1}, 0, \ldots, 0\right]^{t} .
$$

By the definitions of $P$ and $S_{j}$, we have

$$
\Delta_{n} P-\sum_{j=1}^{q-1} \Delta_{n}^{j} T_{n} S_{j}=-\frac{1}{2 \pi i} \int_{\Gamma}\left[\Delta_{n}-\sum_{j=1}^{q-1} \frac{\Delta_{n}^{j} T_{n}}{z^{j}}\right] R(z) d z .
$$


But the identity $(* *)$ shows that

$$
\begin{aligned}
\left(\Delta_{n}-\sum_{j=1}^{q-1} \frac{\Delta_{n}^{j} T_{n}}{z^{j}}\right) R(z) & =\left[T-z I+z I-\sum_{j=0}^{q-1} \frac{\Delta_{n}^{j} T_{n}}{z^{j}}\right] R(z) \\
& =\left[T-z I+\sum_{j=0}^{q} \frac{\Delta_{n}^{j}(z I-T)}{z^{j}}+\frac{\Delta_{n}^{q} T}{z^{q}}\right] R(z) \\
& =-\sum_{j=1}^{q-1} \frac{\Delta_{n}^{j}}{z^{j}}+\frac{\Delta_{n}^{q} R(z)}{z^{q-1}}
\end{aligned}
$$

since $\operatorname{TR}(z)=I+z R(z)$. Since 0 lies outside $\Gamma, \int_{\Gamma} \frac{d z}{z^{j}}=0 \quad$ for $j=1, \ldots, q$, so that

$$
\left\|\left(\mathbf{T}_{q}-\mathbf{T}_{q, n}\right) \mathbf{P}_{q} \mathbf{x}\right\|_{\infty}=\left\|\Delta_{n}^{q} y_{1}\right\|,
$$

where $y_{1}=\left(-\frac{1}{2 \pi i} \int_{\Gamma} \frac{R(z)}{z^{q-1}} d z\right) x_{1}$. Now if $\mathbf{x} \in R\left(\mathbf{P}_{q}\right)$, then $x_{1}=P x_{1} \in R(P)$ and since $P$ commutes with $R(z)$, we see that $y_{1} \in R(P)$. Also, $\left\|y_{1}\right\| \leq \frac{\ell(\Gamma) c_{1}\left\|x_{1}\right\|}{2 \pi \epsilon^{q-1}}$ and hence

$$
\left\|\left(\mathbf{T}_{q}-\mathbf{T}_{q, n}\right)_{\mid R\left(\mathbf{P}_{q}\right)}\right\|_{\infty} \leq \frac{\ell(\Gamma) c_{1}}{2 \pi \epsilon^{q-1}}\left\|\left(T-T_{n}\right)^{q} \mid R(P)\right\|
$$

It follows that if one fixes an integer $q \geq 2$, then the results given in Section 2 become available for the operators $\mathbf{T}_{q}$ and $\mathbf{T}_{q, n}$, and accelerated analogues of Theorem 2.3 and Theorem 2.4 would follow immediately. However, the constants appearing in various error estimates will depend on $q$. In order to find the nature of this dependence on $q$, we proceed as follows. It may be mentioned that the use of the norm \|\|$_{\infty}$ on $\mathbf{X}_{q}$ (instead of the commonly used norm \|\|$_{2}$ ) allows us to achieve our aim.

First we consider the invertibility of $\mathbf{T}_{q, n}-z \mathbf{I}_{q}$.

Proposition 3.2. (a) If $z \neq 0$ and $z I-\sum_{j=0}^{q-1} \frac{\Delta_{n}^{j} T_{n}}{z^{j}}$ is invertible in $B L(X)$, then $\mathbf{T}_{q, n}-z \mathbf{I}_{q}$ is invertible in $B L\left(\mathbf{X}_{q}\right)$.

(b) Let $E$ be a closed subset of $\rho(T)$ and $0 \notin E$. Then there is a positive integer $n_{0}$ such that for all $n \geq n_{0}$ and $q=2,3, \ldots$, we have $E \subset \rho\left(\mathbf{T}_{q, n}\right)$.

If, in fact, $\min \{|z|: z \in E\}>1$, then for all $n \geq n_{0}$ and $q=2,3, \ldots$,

$$
\max _{z \in E}\left\|\left(\mathbf{T}_{q}-z \mathbf{I}_{q}\right)^{-1}\right\|_{\infty} \leq C_{1} \quad \text { and } \quad \max _{z \in E}\left\|\left(\mathbf{T}_{q, n}-z \mathbf{I}_{q}\right)^{-1}\right\|_{\infty} \leq C_{2}
$$

for some constants $C_{1}$ and $C_{2}$ independent of $n$ and $q$.

Proof. (a) Let

$$
A_{k, n}(z)=z I-\sum_{j=0}^{k-1} \frac{\Delta_{n}^{j} T_{n}}{z^{j}}, \quad k=1, \ldots, q
$$


For $z \neq 0$, let $B_{q, n}(z)$ denote the inverse of $A_{q, n}(z)$. Then it can be verified by direct multiplication that the inverse of $\mathbf{T}_{q, n}-z \mathbf{I}_{q}$ is given by the $q \times q$ matrix

$$
\left[\begin{array}{cccccc}
-B_{q} & I-B_{q} A_{1} & z\left(I-B_{q} A_{2}\right) & \cdots & \cdots & z^{q-2}\left(I-B_{q} A_{q-1}\right) \\
-\frac{B_{q}}{z} & \frac{-B_{q} A_{1}}{z} & I-B_{q} A_{2} & \cdots & \cdots & z^{q-3}\left(I-B_{q} A_{q-1}\right) \\
\vdots & \vdots & \vdots & \cdots & \vdots & \vdots \\
\frac{-B_{q}}{z^{q-2}} & \frac{-B_{q} A_{1}}{z^{q-2}} & \frac{-B_{q} A_{2}}{z^{q-3}} & \cdots & \frac{-B_{q} A_{q-2}}{z} & I-B_{q} A_{q-1} \\
\frac{-B_{q}}{z^{q-1}} & \frac{-B_{q} A_{1}}{z^{q-1}} & \frac{-B_{q} A_{2}}{z^{q-2}} & \ldots & \frac{-B_{q} A_{q-2}}{z^{2}} & \frac{-B_{q} A_{q-1}}{z}
\end{array}\right]
$$

where we have written $B_{q}$ for $B_{q, n}(z)$ and $A_{k}$ for $A_{k, n}(z)$.

(b) Since $E$ is a closed subset of $\mathbb{C}$ and $0 \notin E, \min \{|z|: z \in E\}=\delta>0$. Since $\left\|T_{n}\right\|$ is bounded and $\max _{z \in E}\|R(z)\|<\infty$, there is some $M \geq 1$ such that

$$
\left(\max _{z \in E}\|R(z)\|\right) \max _{n=1,2, \ldots}\left\{\left\|T_{n}\right\|, \frac{\left\|\Delta_{n} T_{n}\right\|}{\delta}\right\} \leq M
$$

Since $\left\|\Delta_{n}^{2}\right\| \rightarrow 0$ as $n \rightarrow \infty$, there is a positive integer $n_{0}$ such that $\left\|\Delta_{n}^{2}\right\|<\frac{\delta^{2}}{M}$ for all $n \geq n_{0}$. Let $z \in E$ and $n \geq n_{0}$. As $M \geq 1$ and $\left\|\Delta_{n}^{2}\right\|^{1 / 2}<\delta$, we see that $z I-\Delta_{n}$ is invertible.

By the identity $(*)$, we have

$$
A_{q, n}(z)=z\left(z I-\Delta_{n}\right)^{-1}\left(z I-T+\frac{\Delta_{n}^{q} T_{n}}{z^{q}}\right) .
$$

Again, since

$$
\frac{\left\|\Delta_{n}^{q} T_{n}\right\|}{\left|z^{q}\right|} \leq \begin{cases}\left\|T_{n}\right\|\left\|\frac{\Delta_{n}^{2}}{\delta^{2}}\right\|^{q / 2}, & \text { if } q \text { is even } \\ \frac{\left\|\Delta_{n} T_{n}\right\|}{\delta}\left\|\frac{\Delta_{n}^{2}}{\delta^{2}}\right\|^{(q-1) / 2}, & \text { if } q \text { is odd }\end{cases}
$$

it follows that

$$
\left\|R(z) \frac{\Delta_{n}^{q} T_{n}}{z^{q}}\right\|<1
$$

Hence for all $z \in E, n \geq n_{0}$ and $q=2,3, \ldots$, the operators $z I-T+\frac{\Delta_{n}^{q} T_{n}}{z^{q}}$ (and consequently) $A_{q, n}(z)$ are invertible in $B L(X)$. By $(a)$ above, it follows that $E \subset \rho\left(\mathbf{T}_{q, n}\right)$ for all $n \geq n_{0}$ and $q=2,3, \ldots$.

Next, assume that $\delta>1$. Then

$$
\begin{aligned}
\left\|\left(\mathbf{T}_{q}-z \mathbf{I}_{q}\right)^{-1}\right\|_{\infty} & \leq \max \left\{\|R(z)\|, \frac{\|R(z)\|}{|z|^{k}}+\sum_{j=1}^{k} \frac{1}{|z|^{j}}: \quad k=1, \ldots, q-1\right\} \\
& \leq\|R(z)\|+\frac{1}{\delta-1} .
\end{aligned}
$$

Thus

$$
\max _{z \in E}\left\|\left(\mathbf{T}_{q}-z \mathbf{I}_{q}\right)^{-1}\right\|_{\infty} \leq C_{1},
$$

where $C_{1}$ is a constant independent of $q$. 
We have noted earlier that as $n \rightarrow \infty,\left\|\left(\mathbf{T}_{q}-\mathbf{T}_{q, n}\right) \mathbf{T}_{q}\right\|_{\infty}$ and $\left\|\left(\mathbf{T}_{q}-\mathbf{T}_{q, n}\right) \mathbf{T}_{q, n}\right\|_{\infty}$ tend to 0 uniformly in $q=2,3, \ldots$. Hence we can assume, without loss of generality, that for all $n \geq n_{0}$ and $q=2,3, \ldots$,

$$
\left\|\left(\mathbf{T}_{q}-\mathbf{T}_{q, n}\right)^{2}\right\|_{\infty}<\delta^{2} \quad \text { and } \quad C_{1}\left\|\left(\mathbf{T}_{q}-\mathbf{T}_{q, n}\right) \mathbf{T}_{q, n}\right\|_{\infty} \leq \frac{\delta}{2}
$$

By Lemma 2.1 applied to the operators $\mathbf{T}_{q}$ and $\mathbf{T}_{q, n}$, we have

$$
\left\|\left(\mathbf{T}_{q, n}-z \mathbf{I}_{q}\right)^{-1}\right\|_{\infty} \leq 2 C_{1}\left[1+\frac{\left\|\mathbf{T}_{q}-\mathbf{T}_{q, n}\right\|_{\infty}}{\delta}\right] \leq C_{2}, \text { say. }
$$

We remark that the condition $\min \{|z|: z \in E\}>1$ cannot be dropped from part (b) of Proposition 3.2, that is, if $\min \{|z|: z \in E\} \leq 1$, then $\left\|\left(\mathbf{T}_{q}-z \mathbf{I}_{q}\right)^{-1}\right\|_{\infty}$ may tend to infinity as $q \rightarrow \infty$. The simplest example is obtained by letting $X=\mathbb{C}$ and for a fixed $c \in \mathbb{C}$,

$$
T x=c x, \quad x \in X .
$$

Then for $z \in \mathbb{C}$ with $z \neq c$ and $z \neq 0$ and for $x \in X,(T-z I)^{-1} x=x /(c-z)$, so that

$$
\begin{aligned}
\left(\mathbf{T}_{q}-z \mathbf{I}_{q}\right)^{-1}\left[x_{1}, \ldots, x_{q}\right]^{t}= & {\left[\frac{x_{1}}{c-z}, \frac{x_{1}}{z(c-z)}-\frac{x_{2}}{z}, \ldots\right.} \\
& \left.\left(\frac{x_{1}}{z^{q-1}(c-z)}-\frac{x_{2}}{z^{q-1}}-\cdots-\frac{x_{q}}{z}\right)\right]^{t} .
\end{aligned}
$$

Since

$$
\left(\mathbf{T}_{q}-z \mathbf{I}_{q}\right)^{-1}[1,0, \ldots, 0]^{t}=\left[\frac{1}{c-z}, \frac{1}{z(c-z)}, \ldots, \frac{1}{z^{q-1}(c-z)}\right]^{t}
$$

we have

$$
\left\|\left(\mathbf{T}_{q}-z \mathbf{I}_{q}\right)^{-1}\right\|_{\infty} \geq \frac{1}{|z|^{q-1}|c-z|} .
$$

Thus if $|z|<1$, then $\left\|\left(\mathbf{T}_{q}-z \mathbf{I}_{q}\right)^{-1}\right\|_{\infty}$ tends to infinity as $q \rightarrow \infty$.

Taking $E=\Gamma$, we see that for all $n \geq n_{0}$ and $q=2,3, \ldots, \Gamma \subset \rho\left(\mathbf{T}_{q, n}\right)$, so that

$$
\mathbf{P}_{q, n}=-\frac{1}{2 \pi i} \int_{\Gamma}\left(\mathbf{T}_{q, n}-z \mathbf{I}_{q}\right)^{-1} d z
$$

defines the spectral projection associated with $\mathbf{T}_{q, n}$ and $\Lambda_{q, n}=\sigma\left(\mathbf{T}_{q, n}\right) \cap \operatorname{Int} \Gamma$.

Theorem 3.3. If $\min \{|\lambda|: \lambda \in \Lambda\}>1$ and the curve $\Gamma$ lies in $\{z \in \mathbb{C}:|z| \geq \delta\}$, where $\delta>1$, then for all large $n$ and $q=2,3, \ldots$, we have

$$
\max _{z \in \Gamma}\left\|\left(\mathbf{T}_{q}-z \mathbf{I}_{q}\right)^{-1}\right\|_{\infty} \leq C_{1} \quad \text { and } \max _{z \in \Gamma}\left\|\left(\mathbf{T}_{q, n}-z \mathbf{I}_{q}\right)^{-1}\right\|_{\infty} \leq C_{2}
$$

for some constants $C_{1}$ and $C_{2}$, independent of $n$ and $q$. Also,

$$
\begin{aligned}
\left\|\left(\mathbf{T}_{q}-\mathbf{T}_{q, n}\right) \mathbf{P}_{q}\right\|_{\infty} & \leq \frac{\ell(\Gamma)}{2 \pi \delta} C_{1}\left\|\left(\mathbf{T}_{q}-\mathbf{T}_{q, n}\right) \mathbf{T}_{q}\right\|_{\infty}, \\
\left\|\left(\mathbf{P}_{q}-\mathbf{P}_{q, n}\right) \mathbf{P}_{q}\right\|_{\infty} & \leq \frac{\ell(\Gamma)}{2 \pi} C_{1} C_{2}\left\|\left(\mathbf{T}_{q}-\mathbf{T}_{q, n}\right) \mathbf{P}_{q}\right\|_{\infty}, \\
\left\|\left(\mathbf{T}_{q}-\mathbf{T}_{q, n}\right) \mathbf{P}_{q, n}\right\|_{\infty} & \leq \frac{\ell(\Gamma)}{2 \pi \delta} C_{2}\left\|\left(\mathbf{T}_{q}-\mathbf{T}_{q, n}\right) \mathbf{T}_{q, n}\right\|_{\infty}, \\
\left\|\left(\mathbf{P}_{q}-\mathbf{P}_{q, n}\right) \mathbf{P}_{q, n}\right\|_{\infty} & \leq \frac{\ell(\Gamma)}{2 \pi} C_{1} C_{2}\left\|\left(\mathbf{T}_{q}-\mathbf{T}_{q, n}\right) \mathbf{P}_{q, n}\right\|_{\infty}
\end{aligned}
$$


In particular,

$$
\left\|\left(\mathbf{P}_{q}-\mathbf{P}_{q, n}\right) \mathbf{P}_{q}\right\|_{\infty} \rightarrow 0 \quad \text { and } \quad\left\|\left(\mathbf{P}_{q}-\mathbf{P}_{q, n}\right) \mathbf{P}_{q, n}\right\|_{\infty} \rightarrow 0
$$

as $n \rightarrow \infty$, uniformly in $q=2,3, \ldots$.

Proof. By part (b) of Proposition 3.2, we have

$$
\max _{z \in \Gamma}\left\|\left(\mathbf{T}_{q}-z \mathbf{I}_{q}\right)^{-1}\right\|_{\infty} \leq C_{1}, \quad \max _{z \in \Gamma}\left\|\left(\mathbf{T}_{q, n}-z \mathbf{I}_{q}\right)^{-1}\right\|_{\infty} \leq C_{2}
$$

for all $n \geq n_{0}$ and $q=2,3, \ldots$. By using the resolvent identity it follows, again as in the proof of Theorem 3.1 of [9], that

$$
\begin{aligned}
\left(\mathbf{P}_{q}-\mathbf{P}_{q, n}\right) \mathbf{P}_{q} & =-\frac{1}{2 \pi i} \int_{\Gamma}\left(\mathbf{T}_{q, n}-z \mathbf{I}_{q}\right)^{-1}\left(\mathbf{T}_{q, n}-\mathbf{T}_{q}\right) \mathbf{P}_{q}\left(\mathbf{T}_{q}-z \mathbf{I}_{q}\right)^{-1} d z, \\
\left(\mathbf{P}_{q}-\mathbf{P}_{q, n}\right) \mathbf{P}_{q, n} & =-\frac{1}{2 \pi i} \int_{\Gamma}\left(\mathbf{T}_{q}-z \mathbf{I}_{q}\right)^{-1}\left(\mathbf{T}_{q, n}-\mathbf{T}_{q}\right) \mathbf{P}_{q, n}\left(\mathbf{T}_{q, n}-z \mathbf{I}_{q}\right)^{-1} d z .
\end{aligned}
$$

Further,

$$
\begin{aligned}
\left(\mathbf{T}_{q}-\mathbf{T}_{q, n}\right) \mathbf{P}_{q} & =-\frac{1}{2 \pi i} \int_{\Gamma}\left(\mathbf{T}_{q}-\mathbf{T}_{q, n}\right)\left(\mathbf{T}_{q}-z \mathbf{I}_{q}\right)^{-1} d z \\
& =-\frac{1}{2 \pi i} \int_{\Gamma} \frac{1}{z}\left(\mathbf{T}_{q}-\mathbf{T}_{q, n}\right)\left[\mathbf{T}_{q}\left(\mathbf{T}_{q}-z \mathbf{I}_{q}\right)^{-1}-\mathbf{I}_{q}\right] d z
\end{aligned}
$$

and

$$
\begin{aligned}
\left(\mathbf{T}_{q}-\mathbf{T}_{q, n}\right) \mathbf{P}_{q, n} & =-\frac{1}{2 \pi i} \int_{\Gamma}\left(\mathbf{T}_{q}-\mathbf{T}_{q, n}\right)\left(\mathbf{T}_{q, n}-z \mathbf{I}_{q}\right)^{-1} d z \\
& =-\frac{1}{2 \pi i} \int_{\Gamma} \frac{1}{z}\left(\mathbf{T}_{q}-\mathbf{T}_{q, n}\right)\left[\mathbf{T}_{q, n}\left(\mathbf{T}_{q, n}-z \mathbf{I}_{q}\right)^{-1}-\mathbf{I}_{q}\right] d z .
\end{aligned}
$$

The desired results follow by noting that $-\frac{1}{2 \pi i} \int_{\Gamma} \frac{d z}{z}=0$, as $\Gamma$ does not enclose 0 , and $\left\|\left(\mathbf{T}_{q}-\mathbf{T}_{q, n}\right) \mathbf{T}_{q}\right\|_{\infty} \rightarrow 0,\left\|\left(\mathbf{T}_{q}-\mathbf{T}_{q, n}\right) \mathbf{T}_{q, n}\right\|_{\infty} \rightarrow 0$ as $n \rightarrow \infty$ uniformly in $q=2,3, \ldots$.

In order to treat the case when $\min \{|\lambda|: \lambda \in \Lambda\} \leq 1$, we consider a scaling of the operator $T$. Let $\alpha$ be a positive number. Then

$$
\sigma(\alpha T)=\{\alpha \lambda: \lambda \in \sigma(T)\} .
$$

Also, if $\lambda$ is an isolated eigenvalue of $T$ having finite algebraic multiplicity and $P_{\lambda}$ is the corresponding spectral projection, then $\alpha \lambda$ is an eigenvalue of $\alpha T$ with the same spectral projection, since

$$
-\frac{1}{2 \pi i} \int_{\alpha \Gamma}(\alpha T-w I)^{-1} d w=-\frac{1}{2 \pi i} \int_{\Gamma}(T-z I)^{-1} d z=P_{\lambda} .
$$

Let $\mathbf{T}_{q}(\alpha)$ and $\mathbf{T}_{q, n}(\alpha)$ denote the operators obtained by replacing $T$ and $T_{n}$ by $\alpha T$ and $\alpha T_{n}$ in $\mathbf{T}_{q}$ and $\mathbf{T}_{q, n}$, respectively.

Lemma 3.4. For $\alpha>0$, let $D_{q}(\alpha): \mathbf{X}_{q} \rightarrow \mathbf{X}_{q}$ be given by

$$
D_{q}(\alpha)\left[x_{1}, \ldots, x_{q}\right]^{t}=\left[x_{1}, \alpha x_{2}, \ldots, \alpha^{q-1} x_{q}\right]^{t} .
$$

Then

(a) $\mathbf{T}_{q}(\alpha)=\left(D_{q}(\alpha)\right)^{-1}\left(\alpha \mathbf{T}_{q}\right) D_{q}(\alpha), \quad \mathbf{T}_{q, n}(\alpha)=\left(D_{q}(\alpha)\right)^{-1}\left(\alpha \mathbf{T}_{q, n}\right) D_{q}(\alpha)$,

$$
\sigma\left(\mathbf{T}_{q}(\alpha)\right)=\left\{\alpha \lambda: \lambda \in \sigma\left(\mathbf{T}_{q}\right)\right\}, \quad \sigma\left(\mathbf{T}_{q, n}(\alpha)\right)=\left\{\alpha \lambda: \lambda \in \sigma\left(\mathbf{T}_{q, n}\right)\right\} .
$$


(b) Let $\mathbf{P}_{q}(\alpha)$ and $\mathbf{P}_{q, n}(\alpha)$, respectively, denote the spectral projections associated with the operators $\mathbf{T}_{q}(\alpha)$ and $\mathbf{T}_{q, n}(\alpha)$ with respect to the curve $\alpha \Gamma$. Then

(i) $\mathbf{P}_{q}(\alpha)=\left(D_{q}(\alpha)\right)^{-1} \mathbf{P}_{q} D_{q}(\alpha), \mathbf{P}_{q, n}(\alpha)=\left(D_{q}(\alpha)\right)^{-1} \mathbf{P}_{q, n} D_{q}(\alpha)$,

(ii) $\left[x_{1}, \ldots, x_{q}\right]^{t} \in R\left(\mathbf{P}_{q}(\alpha)\right)$ (resp., $R\left(\mathbf{P}_{q, n}(\alpha)\right)$ ) if and only if

$\left[x_{1}, \alpha x_{2}, \ldots, \alpha^{q-1} x_{q}\right]^{t} \in R\left(\mathbf{P}_{q}\right)$ (resp., $\left.R\left(\mathbf{P}_{q, n}\right)\right)$,

(iii) $\operatorname{rank} \mathbf{P}_{q}(\alpha)=\operatorname{rank} \mathbf{P}_{q}, \operatorname{rank} \mathbf{P}_{q, n}(\alpha)=\operatorname{rank} \mathbf{P}_{q, n}$.

Proof. (a) Considering the $q \times q$ matrix representing the operator $\mathbf{T}_{q}$ and the $q \times q$ diagonal matrix diag $\left(1, \alpha, \ldots, \alpha^{q-1}\right)$ representing the operator $D_{q}(\alpha)$, we obtain $\mathbf{T}_{q}(\alpha)=\left(D_{q}(\alpha)\right)^{-1}\left(\alpha \mathbf{T}_{q}\right) D_{q}(\alpha)$ by direct multiplication. Since $\mathbf{T}_{q}(\alpha)$ and $\alpha \mathbf{T}_{q}$ are thus similar operators, their spectra are identical. The consideration for $\mathbf{T}_{q, n}(\alpha)$ is exactly the same.

(b) We have

$$
\begin{aligned}
\mathbf{P}_{q}(\alpha) & =-\frac{1}{2 \pi i} \int_{\alpha \Gamma}\left(\mathbf{T}_{q}(\alpha)-w \mathbf{I}_{q}\right)^{-1} d w \\
& =-\frac{1}{2 \pi i} \int_{\alpha \Gamma}\left(D_{q}(\alpha)\right)^{-1}\left(\alpha \mathbf{T}_{q}-w \mathbf{I}_{q}\right)^{-1} D_{q}(\alpha) d w \\
& =\left(D_{q}(\alpha)\right)^{-1}\left(-\frac{1}{2 \pi i} \int_{\Gamma}\left(\mathbf{T}_{q}-z \mathbf{I}_{q}\right)^{-1} d z\right) D_{q}(\alpha)=\left(D_{q}(\alpha)\right)^{-1} \mathbf{P}_{q} D_{q}(\alpha) .
\end{aligned}
$$

Now $\mathbf{x}$ belongs to $R\left(\mathbf{P}_{q}(\alpha)\right)$, that is, $\mathbf{P}_{q}(\alpha) \mathbf{x}=\mathbf{x}$ if and only if $\mathbf{P}_{q} D_{q}(\alpha) \mathbf{x}=D_{q}(\alpha) \mathbf{x}$, that is, $D_{q}(\alpha) \mathbf{x}$ belongs to $R\left(\mathbf{P}_{q}\right)$. This implies that $\operatorname{rank} \mathbf{P}_{q}(\alpha)=\operatorname{rank} \mathbf{P}_{q}$. The consideration for $\mathbf{P}_{q, n}(\alpha)$ are exactly the same.

Theorem 3.5. For all large $n$ and all $q=2,3, \ldots$, let

$$
Y_{q, n}=\left\{x_{1} \in X:\left[x_{1}, \ldots, x_{q}\right]^{t} \in R\left(\mathbf{P}_{q, n}\right) \text { for some } x_{2}, \ldots, x_{q} \in X\right\} .
$$

Then

(a) $\operatorname{rank} \mathbf{P}_{q, n}=\operatorname{rank} \mathbf{P}_{q}=\operatorname{rank} P=\operatorname{dim} Y_{q, n}$.

(b) Let $\min \{|\lambda|: \lambda \in \Lambda\}>\epsilon$. Then for all large $n$ and all $q=2,3, \ldots$,

$$
\hat{\delta}\left(R(P), Y_{q, n}\right) \leq \frac{C}{\epsilon^{q-1}} \min \left\{\left\|\left(T-T_{n}\right)^{q} \mid R(P)\right\|,\left\|\left(T-T_{n}\right)^{q} T_{n}\right\|\right\}
$$

for some constant $C$, independent of $n$ and $q$.

Proof. First we consider a special case when $\min \{|\lambda|: \lambda \in \Lambda\}>1$. In that case, we assume $\min \{|z|: z \in \Gamma\}=\delta>1$.

(a) Since

$$
\left\|\left(\mathbf{P}_{q}-\mathbf{P}_{q, n}\right)^{2}\right\|_{\infty} \leq\left\|\left(\mathbf{P}_{q}-\mathbf{P}_{q, n}\right) \mathbf{P}_{q}\right\|_{\infty}+\left\|\left(\mathbf{P}_{q}-\mathbf{P}_{q, n}\right) \mathbf{P}_{q, n}\right\|_{\infty},
$$

it follows from Theorem 3.3 that there is a positive integer $n_{0}$ such that for all $n \geq n_{0}$ and all $q=2,3, \ldots$, we have $\left\|\left(\mathbf{P}_{q}-\mathbf{P}_{q, n}\right)^{2}\right\|_{\infty}<1$, so that $\operatorname{rank} \mathbf{P}_{q, n}=$ $\operatorname{rank} \mathbf{P}_{q}$. We have already noted that $\operatorname{rank} \mathbf{P}_{q}=\operatorname{rank} P$ for all $q$. Next, since $\delta>1$, we note that for $j=1, \ldots, q-1$,

$$
\begin{aligned}
\left\|S_{j}\right\| & =\left\|-\frac{1}{2 \pi i} \int_{\Gamma} \frac{R(z)}{z^{j}} d z\right\| \leq \frac{\ell(\Gamma)}{2 \pi \delta^{j}} \max _{z \in \Gamma}\|R(z)\| \\
& \leq \frac{\ell(\Gamma)}{2 \pi} \max _{z \in \Gamma}\|R(z)\|=c, \text { say. }
\end{aligned}
$$


Thus for $x \in R(P)$, we have

$$
\begin{aligned}
\left\|J_{q} x\right\|_{\infty} & =\max \left\{\|x\|,\left\|S_{1} x\right\|, \ldots,\left\|S_{q-1} x\right\|\right\} \\
& \leq \max \left\{1,\left\|S_{1}\right\|, \ldots,\left\|S_{q-1}\right\|\right\}\|x\| \\
& \leq \max \{1, c\}\|x\| .
\end{aligned}
$$

Let $\phi_{1}, \ldots, \phi_{m}$ be a basis of $R(P)$ and $\phi_{1}^{*}, \ldots, \phi_{m}^{*}$ be the corresponding adjoint basis of $R\left(P^{*}\right)$. For $j=1, \ldots, m$, let $\psi_{q, n, j}$ denote the first component of $\mathbf{P}_{q, n} J_{q} \phi_{j}$. For $k=1, \ldots, m$, we have $K_{q} \phi_{k}^{*}=\left[\phi_{k}^{*}, 0, \ldots, 0\right]^{t}$. Hence

$$
\left\langle\psi_{q, n, j}, \phi_{k}^{*}\right\rangle=\left\langle\mathbf{P}_{q, n} J_{q} \phi_{j}, K_{q} \phi_{k}^{*}\right\rangle
$$

for $j, k,=1, \ldots, m$. Now fix $j, 1 \leq j \leq m$. Then

$$
\begin{aligned}
\left\|\mathbf{P}_{q, n} J_{q} \phi_{j}-J_{q} \phi_{j}\right\|_{\infty} & =\left\|\left(\mathbf{P}_{q, n}-\mathbf{P}_{q}\right) \mathbf{P}_{q} J_{q} \phi_{j}\right\|_{\infty} \\
& \leq\left\|\left(\mathbf{P}_{q, n}-\mathbf{P}_{q}\right) \mathbf{P}_{q}\right\|_{\infty}\left\|J_{q} \phi_{j}\right\|_{\infty} \\
& \leq \max \{1, c\}\left\|\left(\mathbf{P}_{q, n}-\mathbf{P}_{q}\right) \mathbf{P}_{q}\right\|_{\infty}\left\|\phi_{j}\right\| .
\end{aligned}
$$

Since $\left\|\left(\mathbf{P}_{q, n}-\mathbf{P}_{q}\right) \mathbf{P}_{q}\right\|_{\infty} \rightarrow 0$ as $n \rightarrow \infty$ uniformly in $q=2,3, \ldots$, by Theorem 3.3 , we see that for all $k=1, \ldots, m$

$$
\left\langle\psi_{q, n, j}, \phi_{k}^{*}\right\rangle \rightarrow\left\langle J_{q} \phi_{j}, K_{q} \phi_{k}^{*}\right\rangle=\delta_{j, k}
$$

as $n \rightarrow \infty$, uniformly in $q=2,3 \ldots$. This shows that there is a positive integer $n_{0}$ such that for all $n \geq n_{0}$ and all $q=2,3, \ldots$, the $m \times m$ matrix $\left[\left\langle\psi_{q, n, j}, \phi_{k}^{*}\right\rangle\right]$ is nonsingular, and hence, $\left\{\psi_{q, n, 1}, \ldots, \psi_{q, n, m}\right\}$ is a linearly independent subset of $Y_{q, n}$. Thus $\operatorname{dim} Y_{q, n} \geq m$. On the other hand, $\operatorname{dim} Y_{q, n} \leq \operatorname{dim} R\left(\mathbf{P}_{q, n}\right)=\operatorname{rank} P=m$ for all large $n$ and all $q=2,3 \ldots$ Hence, $\operatorname{dim} Y_{q, n}=\operatorname{rank} P=m$.

(b) We have

$$
\delta\left(R(P), Y_{q, n}\right)=\sup \left\{\operatorname{dist}\left(x, Y_{q, n}\right): x \in R(P),\|x\|=1\right\} .
$$

Consider $x \in R(P)$ with $\|x\|=1$ and $J_{q} x=\left[x, S_{1} x, \ldots, S_{q} x\right]^{t}$. If $y_{1} \in Y_{q, n}$, then there is some $\mathbf{y} \in R\left(\mathbf{P}_{q, n}\right)$ with $\mathbf{y}=\left[y_{1}, \ldots, y_{q}\right]$. Since $\left\|x-y_{1}\right\| \leq\left\|J_{q} x-\mathbf{y}\right\|_{\infty}$, we have

$$
\begin{aligned}
\operatorname{dist}\left(x, Y_{q, n}\right) & =\inf \left\{\left\|x-y_{1}\right\|: y_{1} \in Y_{q, n}\right\} \\
& \leq \inf \left\{\left\|J_{q} x-\mathbf{y}\right\|_{\infty}: \mathbf{y} \in R\left(\mathbf{P}_{q, n}\right)\right\} .
\end{aligned}
$$

Let $\mathbf{x}=J_{q} x /\left\|J_{q} x\right\|_{\infty}$, so that $\mathbf{x} \in R\left(\mathbf{P}_{q}\right)$ and $\|\mathbf{x}\|_{\infty}=1$. Thus

$$
\begin{aligned}
\operatorname{dist}\left(x, Y_{q, n}\right) & \leq\left\|J_{q} x\right\|_{\infty} \inf \left\{\|\mathbf{x}-\mathbf{y}\|_{\infty}: \mathbf{y} \in R\left(\mathbf{P}_{q, n}\right)\right\} \\
& \leq\left\|J_{q} x\right\|_{\infty} \delta\left(R\left(\mathbf{P}_{q}\right), R\left(\mathbf{P}_{q, n}\right)\right) \\
& \leq \max \{1, c\} \delta\left(R\left(\mathbf{P}_{q}\right), R\left(\mathbf{P}_{q, n}\right)\right)\|x\|,
\end{aligned}
$$

since $\left\|J_{q} x\right\|_{\infty} \leq \max \{1, c\}\|x\|$, as we have just seen. This implies that

$$
\delta\left(R(P), Y_{q, n}\right) \leq \max \{1, c\} \delta\left(R\left(\mathbf{P}_{q}\right), R\left(\mathbf{P}_{q, n}\right)\right) .
$$

But since $\min \{|\lambda|: \lambda \in \Lambda\}>1$, it follows from Theorem 3.3 that

$$
\delta\left(R\left(\mathbf{P}_{q}\right), R\left(\mathbf{P}_{q, n}\right)\right) \leq\left\|\left(\mathbf{P}_{q}-\mathbf{P}_{q, n}\right) \mathbf{P}_{q}\right\|_{\infty} \rightarrow 0
$$

as $n \rightarrow \infty$ uniformly in $q=2,3 \ldots$. Hence, we can choose $n_{0}$ so large that $\delta\left(R(P), Y_{q, n}\right) \leq 1 / 2$ for all $n \geq n_{0}$ and $q=2,3, \ldots$ Since $\operatorname{dim} Y_{q, n}=\operatorname{dim} R(P)$, we have

$$
\delta\left(Y_{q, n}, R(P)\right) \leq \frac{\delta\left(R(P), Y_{q, n}\right)}{1-\delta\left(R(P), Y_{q, n}\right)} \leq 2 \delta\left(R(P), Y_{q, n}\right)
$$


(see [8], p. 264-269). Thus

$$
\begin{aligned}
\hat{\delta}\left(R(P), Y_{q, n}\right) & =\max \left\{\delta\left(R(P), Y_{q, n}\right), \delta\left(Y_{q, n}, R(P)\right)\right\} \\
& \leq 2 \delta\left(R(P), Y_{q, n}\right) \\
& \leq 2 \max \{1, c\} \delta\left(R\left(\mathbf{P}_{q}\right), R\left(\mathbf{P}_{q, n}\right)\right) \\
& \leq 2 \max \{1, c\} \hat{\delta}\left(R\left(\mathbf{P}_{q}\right), R\left(\mathbf{P}_{q, n}\right)\right) .
\end{aligned}
$$

Since $\left\|\mathbf{T}_{q, n}\right\|_{\infty}$ is bounded in $q$ and $n$, and

$$
\left\|\left(\mathbf{T}_{q}-\mathbf{T}_{q, n}\right) \mathbf{T}_{q}\right\|_{\infty} \rightarrow 0, \quad\left\|\left(\mathbf{T}_{q}-\mathbf{T}_{q, n}\right) \mathbf{T}_{q, n}\right\|_{\infty} \rightarrow 0
$$

as $n \rightarrow \infty$ uniformly in $q$, Theorem 2.2 applied to the operators $\mathbf{T}_{q}$ and $\mathbf{T}_{q, n}$ shows that

$$
\begin{aligned}
\hat{\delta}\left(R\left(\mathbf{P}_{q}\right), R\left(\mathbf{P}_{q, n}\right)\right) \leq \frac{\ell(\Gamma)}{\pi} C_{1} C_{2} \min \{ & \left\|\left(\mathbf{T}_{q}-\mathbf{T}_{q, n}\right)_{\mid R\left(\mathbf{P}_{q}\right)}\right\|_{\infty}, \\
& \left.\left\|\left(\mathbf{T}_{q}-\mathbf{T}_{q, n}\right)_{\mid R\left(\mathbf{P}_{q, n}\right)}\right\|_{\infty}\right\},
\end{aligned}
$$

where $\sup _{z \in \Gamma}\left\|\left(\mathbf{T}_{q}-z \mathbf{I}_{q}\right)^{-1}\right\|_{\infty} \leq C_{1}$ and $\sup _{z \in \Gamma}\left\|\left(\mathbf{T}_{q, n}-z \mathbf{I}_{q}\right)^{-1}\right\|_{\infty} \leq C_{2}$. But by Proposition 3.1 with $\epsilon=1$, we have

$$
\left\|\left(\mathbf{T}_{q}-\mathbf{T}_{q, n}\right)_{\mid R\left(\mathbf{P}_{q}\right)}\right\|_{\infty} \leq \frac{\ell(\Gamma) c_{1}}{2 \pi}\left\|\left(T-T_{n}\right)^{q} \mid R(P)\right\| .
$$

Also,

$$
\begin{aligned}
\left.\|\left(\mathbf{T}_{q}-\mathbf{T}_{q, n}\right)_{\mid R\left(\mathbf{P}_{q, n}\right)}\right) \|_{\infty} & \leq\left\|\left(\mathbf{T}_{q}-\mathbf{T}_{q, n}\right) \mathbf{P}_{q, n}\right\|_{\infty} \\
& \leq \frac{\ell(\Gamma)}{2 \pi \delta} C_{2}\left\|\left(\mathbf{T}_{q}-\mathbf{T}_{q, n}\right) \mathbf{T}_{q, n}\right\|_{\infty} \\
& \leq \frac{\ell(\Gamma)}{2 \pi \delta} C_{2}\left\|\Delta_{n}^{q} T_{n}\right\|,
\end{aligned}
$$

as we have already seen. (Note that $\min \{|z|: z \in \Gamma\}=\delta>1$.) Thus

$$
\hat{\delta}\left(R(P), Y_{q, n}\right) \leq C \min \left\{\left\|\left(T-T_{n}\right)_{\mid R(P)}^{q}\right\|,\left\|\left(T-T_{n}\right)^{q} T_{n}\right\|\right\}
$$

for all large $n, q=2,3, \ldots$ and some constant $C$, independent of $n$ and $q$.

Finally, we consider the general case when $\min \{|\lambda|: \lambda \in \Lambda\}>\epsilon$. We choose $\alpha=1 / \epsilon$ and consider the scaled operators $\mathbf{T}_{q}(\alpha), \mathbf{P}_{q}(\alpha), \mathbf{T}_{q, n}(\alpha)$ and $\mathbf{P}_{q, n}(\alpha)$. By what we have just proved and by Lemma 3.4,

$$
\operatorname{rank} \mathbf{P}_{q, n}=\operatorname{rank} \mathbf{P}_{q, n}(\alpha)=\operatorname{rank} \mathbf{P}_{q}(\alpha)=\operatorname{rank} \mathbf{P}_{q}
$$

for all large $n$ and $q=2,3 \ldots$ Also, $P(\alpha)=P$ and the first components of the elements of $R\left(\mathbf{P}_{q, n}(\alpha)\right)$ and $R\left(\mathbf{P}_{q, n}\right)$ are the same, that is, $Y_{q, n}(\alpha)=Y_{q, n}$. Hence there is a constant $C(\alpha)$ independent of $n$ and $q$ such that

$$
\begin{aligned}
\hat{\delta}\left(R(P), Y_{q, n}\right) & =\hat{\delta}\left(R(P(\alpha)), Y_{q, n}(\alpha)\right) \\
& \leq C(\alpha) \min \left\{\left\|\left(\alpha T-\alpha T_{n}\right)^{q} \mid R(P(\alpha))\right\|,\left\|\left(\alpha T-\alpha T_{n}\right)^{q} \alpha T_{n}\right\|\right\} \\
& =\frac{\max \left\{\alpha, \alpha^{2}\right\} C(\alpha)}{\epsilon^{q-1}} \min \left\{\left\|\left(T-T_{n}\right)^{q} \mid R(P)\right\|,\left\|\left(T-T_{n}\right)^{q} T_{n}\right\|\right\} .
\end{aligned}
$$

Hence the result.

Recall that $\Lambda=\left\{\lambda_{1}, \ldots, \lambda_{m}\right\}$, where each $\lambda_{j}, j=1, \ldots, m$, is an eigenvalue of $T$ counted according to its algebraic multiplicity and

$$
\hat{\lambda}=\frac{\lambda_{1}+\ldots+\lambda_{m}}{m} \text {. }
$$


Since rank $\mathbf{P}_{q, n}=m$ for all large $n$ and all $q=2,3, \ldots$,

$$
\sigma\left(\mathbf{T}_{q, n}\right) \cap \operatorname{Int} \Gamma=\left\{\lambda_{q, n, 1}, \ldots, \lambda_{q, n, m}\right\},
$$

where each $\lambda_{q, n, j}, j=1, \ldots, m$, is an eigenvalue of $\mathbf{T}_{q, n}$ counted according to its algebraic multiplicity.

These are the eigenvalues obtained from $q$ th spectral analysis of $T$. In the words of Dellwo and Friedman, they comprise the legitimate portion of the $q$ th order approximate spectra associated with $\Lambda$.

Let

$$
\hat{\lambda}_{q, n}=\frac{\lambda_{q, n, 1}+\cdots+\lambda_{q, n, m}}{m} .
$$

Theorem 3.6. Let $\min \{|\lambda|: \lambda \in \Lambda\}>\epsilon$. Then for all large $n$ and all $q=2,3, \ldots$,

$$
\left|\hat{\lambda}-\hat{\lambda}_{q, n}\right| \leq \frac{C}{\epsilon^{q-1}} \min \left\{\left\|\left(T-T_{n}\right)^{q} \mid R(P)\right\|,\left\|\left(T-T_{n}\right)^{q} T_{n}\right\|\right\}
$$

for some constant $C$, independent of $n$ and $q$.

If $\Lambda=\{\lambda\}$ and the ascent of $\lambda$ as an eigenvalue of $T$ is $l$, then for each $i=$ $1, \ldots, m$, we have

$$
\left|\lambda-\lambda_{q, n, i}\right|^{l} \leq \frac{C^{\prime}}{\epsilon^{q-1}} \min \left\{\left\|\left(T-T_{n}\right)^{q} \mid R(P)\right\|,\left\|\left(T-T_{n}\right)^{q} T_{n}\right\|\right\}
$$

for some constant $C^{\prime}$, independent of $n$ and $q$.

Proof. First we consider a special case when $\min \{|\lambda|: \lambda \in \Lambda\}>1$. By Theorem 3.3 , there is some $n_{0}$ such that for all $n \geq n_{0}$ and all $q=2,3, \ldots$, we have $\left\|\left(\mathbf{P}_{q}-\mathbf{P}_{q, n}\right) \mathbf{P}_{q}\right\|_{\infty} \leq 1 / 2$. It follows that for all such $n$ and $q=2,3, \ldots$, the map $\mathbf{A}_{q, n}$ from $R\left(\mathbf{P}_{q}\right)$ to $R\left(\mathbf{P}_{q, n}\right)$ given by $\mathbf{x} \longmapsto \mathbf{P}_{q, n} \mathbf{x}$ is an isomorphism and if $\mathbf{A}_{q, n}^{-1}$ denotes the inverse map from $R\left(\mathbf{P}_{q, n}\right)$ to $R\left(\mathbf{P}_{q}\right)$, then $\left\|\mathbf{A}_{q, n}^{-1}\right\|_{\infty} \leq 2$. The same argument also shows that the map $\mathbf{B}_{q, n}$ from $R\left(\mathbf{P}_{q, n}\right)$ to $R\left(\mathbf{P}_{q}\right)$ given by $\mathbf{x} \longmapsto \mathbf{P}_{q} \mathbf{x}$ is an isomorphism and $\left\|\mathbf{B}_{q, n}^{-1}\right\|_{\infty} \leq 2$. We choose $\Gamma$ so that $\min \{|z|: z \in \Gamma\}=\delta>1$. Then by Proposition 3.2, we have

$$
\left\|\mathbf{P}_{q}\right\|_{\infty} \leq \frac{\ell(\Gamma)}{2 \pi} C_{1} \quad \text { and } \quad\left\|\mathbf{P}_{q, n}\right\|_{\infty} \leq \frac{\ell(\Gamma)}{2 \pi} C_{2}
$$

where $C_{1}$ and $C_{2}$ are independent of $n$ and $q$. Noting that the algebraic multiplicity of each $\lambda_{i}$ as an eigenvalue of $\mathbf{T}_{q}$ is equal to its algebraic multiplicity as an eigenvalue of $T$, and applying Theorem 2.3 to the operators $\mathbf{T}_{q}$ and $\mathbf{T}_{q, n}$, we have

$$
\begin{aligned}
\left|\hat{\lambda}-\hat{\lambda}_{q, n}\right| \leq 2 \min \left\{\| \mathbf{P}_{q, n}\right. & \left\|_{\infty}\right\|\left(\mathbf{T}_{q}-\mathbf{T}_{q, n}\right)_{\mid R\left(\mathbf{P}_{q}\right)} \|_{\infty}, \\
& \left.\left\|\mathbf{P}_{q}\right\|_{\infty}\left\|\left(\mathbf{T}_{q}-\mathbf{T}_{q, n}\right)_{\mid R\left(\mathbf{P}_{q, n}\right)}\right\|_{\infty}\right\} .
\end{aligned}
$$

But by Proposition 3.1 with $\epsilon=1$, we have

$$
\begin{aligned}
2\left\|\mathbf{P}_{q, n}\right\|_{\infty}\left\|\left(\mathbf{T}_{q}-\mathbf{T}_{q, n}\right)_{\mid R\left(\mathbf{P}_{q}\right)}\right\|_{\infty} & \leq \frac{\ell(\Gamma)}{\pi} C_{2}\left\|\left(\mathbf{T}_{q}-\mathbf{T}_{q, n}\right)_{\mid R\left(\mathbf{P}_{q}\right)}\right\|_{\infty} \\
& \leq \frac{\ell(\Gamma)}{\pi} C_{2} \frac{\ell(\Gamma)}{2 \pi} c_{1}\left\|\left(T-T_{n}\right)^{q} \mid R(P)\right\|,
\end{aligned}
$$

and

$$
\begin{aligned}
2\left\|\mathbf{P}_{q}\right\|_{\infty}\left\|\left(\mathbf{T}_{q}-\mathbf{T}_{q, n}\right)_{\mid R\left(\mathbf{P}_{q, n}\right)}\right\|_{\infty} & \leq \frac{\ell(\Gamma)}{\pi} C_{1}\left\|\left(\mathbf{T}_{q}-\mathbf{T}_{q, n}\right)_{\mid R\left(\mathbf{P}_{q, n}\right)}\right\|_{\infty} \\
& \leq \frac{\ell(\Gamma)}{\pi} C_{1} \frac{\ell(\Gamma)}{2 \pi \delta} C_{2}\left\|\left(T-T_{n}\right)^{q} T_{n}\right\|,
\end{aligned}
$$


as in the proof of Theorem 3.5(b). Thus

$$
\left|\hat{\lambda}-\hat{\lambda}_{q, n}\right| \leq C \min \left\{\left\|\left(T-T_{n}\right)^{q}{ }_{\mid R(P)}\right\|,\left\|\left(T-T_{n}\right)^{q} T_{n}\right\|\right\}
$$

for all large $n, q=2,3, \ldots$ and some constant $C$, independent of $n$ and $q$.

Now let $\Lambda=\{\lambda\}$, so that $\hat{\lambda}=\lambda$. We have noted that the ascent of $\lambda$ as an eigenvalue of $\mathbf{T}_{q}$ is equal to its ascent as an eigenvalue of $T$, namely $l$. Again, applying Theorem 2.3 to the operators $\mathbf{T}_{q}$ and $\mathbf{T}_{q, n}$, we have for $j=1, \ldots, m$,

$$
\begin{aligned}
\left|\lambda-\lambda_{q, n, j}\right|^{l} \leq 2 \min \left\{C_{q, n}\left\|\mathbf{P}_{q, n}\right\|_{\infty}\left\|\left(\mathbf{T}_{q}-\mathbf{T}_{q, n}\right)_{\mid R\left(\mathbf{P}_{q}\right)}\right\|_{\infty},\right. \\
\left.D_{q, n}\left\|\mathbf{P}_{q}\right\|_{\infty}\left\|\left(\mathbf{T}_{q}-\mathbf{T}_{q, n}\right)_{\mid R\left(\mathbf{P}_{q, n}\right)}\right\|_{\infty}\right\},
\end{aligned}
$$

where

$$
\begin{aligned}
C_{q, n} & =\sum_{k=0}^{l-1}\left\|\lambda \mathbf{I}_{q \mid R\left(\mathbf{P}_{q}\right)}-\mathbf{A}_{q, n}^{-1} \mathbf{T}_{q, n} \mathbf{A}_{q, n}\right\|_{\infty}^{l-1-k}\left\|\lambda \mathbf{I}_{q \mid R\left(\mathbf{P}_{q}\right)}-\mathbf{T}_{q \mid R\left(\mathbf{P}_{q}\right)}\right\|_{\infty}^{k} \\
D_{q, n} & =\sum_{k=0}^{l-1}\left\|\lambda \mathbf{I}_{q \mid R\left(\mathbf{P}_{q, n}\right)}-\mathbf{T}_{q, n \mid R\left(\mathbf{P}_{q, n}\right)}\right\|_{\infty}^{l-1-k}\left\|\lambda \mathbf{I}_{q \mid R\left(\mathbf{P}_{q, n}\right)}-\mathbf{B}_{q, n}^{-1} \mathbf{T}_{q} \mathbf{B}_{q, n}\right\|_{\infty}^{k}
\end{aligned}
$$

Note that $\min \{|z|: z \in \Gamma\}=\delta>1$. Therefore, for $j=1, \ldots, m$ we have

$$
\left|\lambda-\lambda_{q, n, j}\right|^{l} \leq C^{\prime} \min \left\{\left\|\left(T-T_{n}\right)^{q} \mid R(P)\right\|,\left\|\left(T-T_{n}\right)^{q} T_{n}\right\|\right\}
$$

for all large $n, q=2,3, \ldots$ and some constant $C^{\prime}$, independent of $n$ and $q$.

Finally, we consider the general when case $\min \{|\lambda|: \lambda \in \Lambda\}>\epsilon$. We choose $\alpha=1 / \epsilon$ and consider the scaled operators $\mathbf{T}_{q}(\alpha)$ and $\mathbf{T}_{q, n}(\alpha)$. By Lemma 3.4, we find that $\alpha \lambda_{1}, \ldots, \alpha \lambda_{m}$ are the eigenvalues of $\mathbf{T}_{q}(\alpha)$ inside the curve $\alpha \Gamma$ and $\alpha \lambda_{q, n, 1}, \ldots, \alpha \lambda_{q, n, m}$ are the eigenvalues of $\mathbf{T}_{q, n}(\alpha)$ inside the curve $\alpha \Gamma$, counted according to their algebraic multiplicities. By what we have just proved, there is some constant $C(\alpha)$, independent of $n$ and $q$ such that

$$
\left|\alpha \hat{\lambda}-\alpha \hat{\lambda}_{q, n}\right| \leq C(\alpha) \min \left\{\left\|\left(\alpha T-\alpha T_{n}\right)^{q} \mid R(P)\right\|,\left\|\left(\alpha T-\alpha T_{n}\right)^{q} \alpha T_{n}\right\|\right\},
$$

so that

$$
\left|\hat{\lambda}-\hat{\lambda}_{q, n}\right| \leq \frac{\max \{1, \alpha\} C(\alpha)}{\epsilon^{q-1}} \min \left\{\left\|\left(T-T_{n}\right)^{q} \mid R(P)\right\|,\left\|\left(T-T_{n}\right)^{q} T_{n}\right\|\right\},
$$

as desired. If $\Lambda=\{\lambda\}$, then the ascent of $\alpha \lambda$ as an eigenvalue of $\mathbf{T}_{q}(\alpha)$ equals $l$. Hence the estimates for $\left|\lambda-\lambda_{q, n, j}\right|^{l}, j=1, \ldots, m$, follow similarly.

If $\Lambda=\{\lambda\}$ with $|\lambda|>\epsilon$ and $f$ is an analytic function in the neighborhood of $\lambda$, then by the functional calculus it is easy to see that for all large $n$ and $q=2,3, \ldots$,

$$
\left|f(\lambda)-\frac{1}{m} \sum_{j=1}^{m} f\left(\lambda_{q, n, j}\right)\right| \leq \frac{C}{\epsilon^{q-1}} \min \left\{\left\|\left(T-T_{n}\right)^{q} \mid R(P)\right\|,\left\|\left(T-T_{n}\right)^{q} T_{n}\right\|\right\},
$$

where $C$ is a constant independent of $n$ and $q$. The above-mentioned estimate is, in fact, an accelerated analogue of a result of Descloux, Nassif and Rappaz ([6], [7]).

Next, we consider approximation of an element of $R(P)$ by an eigenvector obtained from a higher order spectral analysis of $T$. 
Theorem 3.7. Let $\mathbf{x}=\left[x_{1}, \ldots, x_{q}\right]^{t}$ be an eigenvector of $\mathbf{T}_{q, n}$ corresponding to an eigenvalue in $\Lambda_{q, n}$ such that $\left\|x_{1}\right\|=1$. If $\min \{|\lambda|: \lambda \in \Lambda\}>\epsilon$, then there is a constant $C$ such that

$$
\left\|x_{1}-P x_{1}\right\| \leq \frac{C}{\epsilon^{q-1}}\left\|\left(T-T_{n}\right)^{q} T_{n}\right\|
$$

for all large $n$ and $q=2,3, \ldots$.

Proof. Since $\mathbf{P}_{q} \mathbf{x}=\left[P x_{1}, S_{1} x_{1}, \ldots, S_{q-1} x_{1}\right]^{t}$ and $\mathbf{P}_{q, n} \mathbf{x}=\mathbf{x}$, we have

$$
\left\|x_{1}-P x_{1}\right\| \leq\left\|\mathbf{x}-\mathbf{P}_{q} \mathbf{x}\right\|_{\infty}=\left\|\left(\mathbf{P}_{q, n}-\mathbf{P}_{q}\right) \mathbf{P}_{q, n} \mathbf{x}\right\|_{\infty}
$$

If $\min \{|\lambda|: \lambda \in \Lambda\}>1$, then $\|\mathbf{x}\|_{\infty}=\left\|x_{1}\right\|=1$ and hence Theorem 3.3 shows there is a constant $C$ with

$$
\left\|x_{1}-P x_{1}\right\| \leq\left\|\left(\mathbf{P}_{q, n}-\mathbf{P}_{q}\right) \mathbf{P}_{q, n}\right\|_{\infty} \leq C\left\|\left(\mathbf{T}_{q, n}-\mathbf{T}_{q}\right) \mathbf{T}_{q, n}\right\|_{\infty}=C\left\|\left(T-T_{n}\right)^{q} T_{n}\right\| .
$$

If $\min \{|\lambda|: \lambda \in \Lambda\}>\epsilon$, then, as before, we choose $\alpha=1 / \epsilon$ and consider the scaled operators $\mathbf{T}_{q, n}(\alpha), \mathbf{P}_{q, n}(\alpha), \mathbf{T}_{q}(\alpha)$ and $\mathbf{P}_{q}(\alpha)$. Since $P(\alpha)=P$ and the first components of the elements of $R\left(\mathbf{P}_{q, n}(\alpha)\right)$ and $R\left(\mathbf{P}_{q, n}\right)$ are the same, there is a constant $C(\alpha)$ such that

$$
\left\|x_{1}-P x_{1}\right\| \leq C(\alpha)\left\|\left(\alpha T-\alpha T_{n}\right)^{q} \alpha T_{n}\right\|=\frac{\alpha^{2} C(\alpha)}{\epsilon^{q-1}}\left\|\left(T-T_{n}\right)^{q} T_{n}\right\| .
$$

The result in Theorem 3.7 was also obtained by Dellwo and Friedman in [5].

Let $\Lambda=\{\lambda\}$ and $l$ be the ascent of $\lambda$. Let $\lambda_{q, n} \in \Lambda_{q, n}$ such that $\lambda_{q, n} \rightarrow \lambda$ as $n \rightarrow \infty$ uniformly in $q=2,3, \ldots$.

Theorem 3.8. Let $|\lambda|>\epsilon$. Suppose that

$$
\mathbf{w}_{q, n}=\left[w_{q, n}, \frac{w_{q, n}}{\lambda_{q, n}}, \ldots, \frac{w_{q, n}}{\left(\lambda_{q, n}\right)^{q-1}}\right]^{t} \in N\left(\mathbf{T}_{q, n}-\lambda_{q, n} \mathbf{I}_{q}\right)
$$

with $\left\|w_{q, n}\right\|=1$. Then there is some $u_{q, n} \in N(T-\lambda I)$ such that

$$
\left\|w_{q, n}-u_{q, n}\right\| \leq C\left\{\frac{1}{\epsilon^{q-1}}\left\|\left(T-T_{n}\right)^{q} \mid R(P)\right\|\right\}^{1 / l}
$$

for all $q=2,3, \ldots$, where $C$ is a constant independent of $q$ and $n$.

Proof. Consider the particular case when $|\lambda|>1$. Then for all large $n$ and $q=$ $2,3, \ldots,\left|\lambda_{q, n}\right|>1$. Thus $\left\|\mathbf{w}_{q, n}\right\|_{\infty}=\left\|w_{q, n}\right\|=1$. Applying Theorem 2.4 to the operators $\mathbf{T}_{q}$ and $\mathbf{T}_{q, n}$, we obtain some $\mathbf{u}_{q, n} \in N\left(\mathbf{T}_{q}-\lambda \mathbf{I}_{q}\right)$ such that

$$
\left\|\mathbf{w}_{q, n}-\mathbf{u}_{q, n}\right\|_{\infty} \leq C\left\{\left\|\left(\mathbf{T}_{q}-\mathbf{T}_{q, n}\right)_{\mid R\left(\mathbf{P}_{q}\right)}\right\|_{\infty}\right\}^{1 / l}
$$

Since $\mathbf{u}_{q, n}=J_{q} u_{q, n}$ for some $u_{q, n} \in N(T-\lambda I$ ), Proposition 3.1 (with $\epsilon=1$ ) shows that

$$
\left\|w_{q, n}-u_{q, n}\right\| \leq\left\|\mathbf{w}_{q, n}-\mathbf{u}_{q, n}\right\|_{\infty} \leq C^{\prime}\left\{\left\|\left(T-T_{n}\right)_{\mid R(P)}^{q}\right\|\right\}^{1 / l}
$$

for some constant $C^{\prime}$, independent of $n$ and $q$. 
Finally, to treat the general case, we choose $\alpha=1 / \epsilon$ and consider the scaled operators $\mathbf{T}_{q}(\alpha), \mathbf{P}_{q}(\alpha), \mathbf{T}_{q, n}(\alpha)$ and $\mathbf{P}_{q, n}(\alpha)$. Since $P(\alpha)=P$ and the first components of the elements of $R\left(\mathbf{P}_{q, n}(\alpha)\right)$ and $R\left(\mathbf{P}_{q, n}\right)$ are the same, there is a constant $C^{\prime}(\alpha)$ such that

$$
\begin{aligned}
\left\|u_{q, n}-w_{q, n}\right\| & \leq C^{\prime}(\alpha)\left\{\left\|\left(\alpha T-\alpha T_{n}\right)_{\mid R(P)}^{q}\right\|\right\}^{1 / l} \\
& =C^{\prime}(\alpha)\left\{\frac{1}{\epsilon^{q}}\left\|\left(T-T_{n}\right)^{q} \mid R(P)\right\|\right\}^{1 / l} .
\end{aligned}
$$

Hence the result follows.

\section{NUMERICAL EXAMPLES}

Let $\lambda$ be a nonzero defective eigenvalue of $T$ of algebraic multiplicity $m$ and ascent $l>1$. We illustrate by numerical examples how the weighted arithmetic mean $\hat{\lambda}_{q, n}$ gives a better approximation than do the individual eigenvalues $\lambda_{q, n, 1}, \ldots, \lambda_{q, n, m}$, provided by the $q$ th order spectral analysis when $q=2,3,4,5,6$.

Let $X=C([a, b])$ and $T$ be an integral operator given by

$$
T x(s)=\int_{a}^{b} k(s, t) x(t) d t, \quad x \in X, \quad s \in[a, b],
$$

where the kernel $k$ is continuous on $[a, b] \times[a, b]$. In actual computations, $T$ is replaced by its approximation $\tilde{T}$ given by

$$
\tilde{T} x(s)=\sum_{j=1}^{M} w_{j}^{(M)} k\left(s, t_{j}^{(M)}\right) x\left(t_{j}^{(M)}\right), \quad x \in X, \quad s \in[a, b],
$$

where $M$ is very large. Here the nodes $t_{1}^{(M)}, \ldots, t_{M}^{(M)}$ in $[a, b]$ and the weights $w_{1}^{(M)}, \ldots, w_{M}^{(M)}$ in $\mathbb{C}$ give a convergent quadrature formula

$$
Q x=\sum_{j=1}^{M} w_{j}^{(M)} x\left(t_{j}^{(M)}\right), \quad x \in X .
$$

Consider a finite rank operator $T_{n}$ given by

$$
T_{n} x=\sum_{j=1}^{n}\left\langle x, x_{j}^{*}\right\rangle x_{j}, \quad x \in X
$$

where $x_{1}, \ldots, x_{n}$ are in $X$ and $x_{1}^{*}, \ldots, x_{n}^{*}$ are in $X^{*}$. Then the eigenvalue problem for $\mathbf{T}_{q, n}$ can be reduced to an eigenvalue problem for the matrix $\mathbf{A}_{q, n}$ where

$$
\mathbf{A}_{q, n}=\left[\begin{array}{ccccc}
A_{n}^{(0)} & A_{n}^{(1)} & \ldots & \ldots & A_{n}^{(q-1)} \\
I_{n} & 0 & \cdots & \cdots & 0 \\
0 & \ddots & & & \vdots \\
\vdots & & \ddots & & \vdots \\
0 & 0 & \cdots & I_{n} & 0
\end{array}\right]
$$

Here $A_{n}^{(k)}=\left[\left\langle\Delta_{n}^{k} x_{j}, x_{i}^{*}\right\rangle\right]$ for $k=0,1, \ldots, q-1$ and $I_{n}$ is the $n \times n$ identity matrix. 
Note that this matrix eigenvalue problem is of size $n q$. If $\left\|T-T_{n}\right\| \rightarrow 0$, then both $\left\|\left(T-T_{n}\right)^{q} \mid R(P)\right\|$ and $\left\|\left(T-T_{n}\right)^{q} T_{n}\right\|$ are less than or equal to a constant times $\left\|T-T_{n}\right\|^{q}$. Thus if we keep $n$ fixed and increase the order $q$ of the spectral analysis, then the size of the matrix eigenvalue problem increases arithmetically with respect to $q$ while the accuracy of the spectral approximation increases geometrically.

Example. Let $a=0, b=1$ and

$$
k(s, t)=\left\{\begin{array}{lll}
s-t / 2, & \text { if } & 0 \leq s \leq t \leq 1 \\
t / 2, & \text { if } & 0 \leq t<s \leq 1
\end{array}\right.
$$

Then each $\lambda_{j}=\frac{1}{(2 j-1)^{2} \pi^{2}}, j=1,2, \ldots$, is an eigenvalue of $T$ of algebraic multiplicity $m=2$ and ascent $l=2$. We have chosen the nodes and weights as follows:

$$
t_{i}^{(M)}= \begin{cases}\frac{i-1 / \sqrt{3}}{M}, & \text { if } i \text { is odd } \\ \frac{i-1+1 / \sqrt{3}}{M}, & \text { if } i \text { is even }\end{cases}
$$

and

$$
w_{i}^{(M)}=\frac{1}{M}, \quad i=1, \ldots, M
$$

These are obtained by the compound Gauss Two Point Rule on $[0,1]$.

For $n \ll M$, let $w_{1}^{(n)}, \ldots, w_{n}^{(n)}$ and $t_{1}^{(n)}, \ldots, t_{n}^{(n)}$ be the weights and the nodes, respectively, associated with the compound Gauss Two Point Rule on [0,1]. Let $e_{1}^{(n)}, \ldots, e_{n}^{(n)}$ be the hat functions corresponding to the nodes $t_{1}^{(n)}, \ldots, t_{n}^{(n)}$. We consider the following two approximations of the integral operator $T$.

i) Nyström Approximation:

$$
T_{n}^{N} x(s)=\sum_{j=1}^{n} w_{j}^{(n)} k\left(s, t_{j}^{(n)}\right) x\left(t_{j}^{(n)}\right), \quad x \in C([0,1]), \quad s \in[0,1] .
$$

ii) Projection Approximation:

$$
T_{n}^{P}=\pi_{n} T, \text { where } \pi_{n} x=\sum_{j=1}^{n} x\left(t_{j}^{(n)}\right) e_{j}^{(n)}, \quad x \in C([0,1]) .
$$

Note that the sequence $\left(T_{n}^{N}\right)$ satisfies the hypotheses (H1) and (H2), but $\left\|T-T_{n}^{N}\right\|$ does not tend to 0 as $n \rightarrow \infty$ (cf. [3], p. 197). On the other hand, $\left\|T-T_{n}^{P}\right\| \rightarrow 0$ as $n \rightarrow \infty$ (cf. Theorem 4.5 of [3]).

Let $\tilde{\lambda}$ denote the arithmetic mean of the two eigenvalues of $\tilde{T}$ which are close to the largest eigenvalue $\lambda=1 / \pi^{2}$ of $T$. Also, let $\lambda_{q, n, 1}$ and $\lambda_{q, n, 2}$ denote the eigenvalues provided by the $q$ th order spectral analysis which are close to $\tilde{\lambda}$, and $\hat{\lambda}_{q, n}=\frac{\lambda_{q, n, 1}+\lambda_{q, n, 2}}{2}$. We have taken $M=500$ and $n=10,20,30,40$. The following computations were performed on HP9000/700 model J200 in single precision with an accuracy of 7 digits and in double precision with an accuracy of 15 digits. These numerical results illustrate that, in general, the rate of convergence of the $\hat{\lambda}_{q, n}$ to $\tilde{\lambda}$ is faster than that of the individual eigenvalues $\lambda_{q, n, 1}$ and $\lambda_{q, n, 2}$. 


\section{Calculations in Single Precision}

TABLe 4.1. $q=2$,

Nyström Approximation

\begin{tabular}{|l|c|c|c|}
\hline$n$ & $\left|\tilde{\lambda}-\lambda_{q, n, 1}\right|$ & $\left|\tilde{\lambda}-\lambda_{q, n, 2}\right|$ & $\left|\tilde{\lambda}-\hat{\lambda}_{q, n}\right|$ \\
\hline 10 & $8.96 \times 10^{-4}$ & $9.50 \times 10^{-4}$ & $9.23 \times 10^{-4}$ \\
20 & $2.15 \times 10^{-4}$ & $2.36 \times 10^{-4}$ & $2.25 \times 10^{-4}$ \\
30 & $1.02 \times 10^{-4}$ & $1.02 \times 10^{-4}$ & $9.98 \times 10^{-5}$ \\
40 & $1.44 \times 10^{-5}$ & $9.78 \times 10^{-5}$ & $5.61 \times 10^{-5}$ \\
\hline
\end{tabular}

TABLE 4.3. $q=3$,

Nyström Approximation

\begin{tabular}{|l|c|c|c|}
\hline$n$ & $\left|\tilde{\lambda}-\lambda_{q, n, 1}\right|$ & $\left|\tilde{\lambda}-\lambda_{q, n, 2}\right|$ & $\left|\tilde{\lambda}-\hat{\lambda}_{q, n}\right|$ \\
\hline 10 & $6.69 \times 10^{-5}$ & $6.69 \times 10^{-5}$ & $1.37 \times 10^{-5}$ \\
20 & $3.25 \times 10^{-5}$ & $3.25 \times 10^{-5}$ & $8.94 \times 10^{-7}$ \\
30 & $3.93 \times 10^{-5}$ & $3.87 \times 10^{-5}$ & $3.05 \times 10^{-7}$ \\
40 & $4.67 \times 10^{-5}$ & $4.67 \times 10^{-5}$ & $1.04 \times 10^{-7}$ \\
\hline
\end{tabular}

TABLE 4.5. $q=4$,

Nyström Approximation

\begin{tabular}{|l|c|c|c|}
\hline$n$ & $\left|\tilde{\lambda}-\lambda_{q, n, 1}\right|$ & $\left|\tilde{\lambda}-\lambda_{q, n, 2}\right|$ & $\left|\tilde{\lambda}-\hat{\lambda}_{q, n}\right|$ \\
\hline 10 & $2.65 \times 10^{-5}$ & $4.27 \times 10^{-5}$ & $8.08 \times 10^{-6}$ \\
20 & $3.31 \times 10^{-5}$ & $3.31 \times 10^{-5}$ & $4.10 \times 10^{-7}$ \\
30 & $3.95 \times 10^{-5}$ & $3.95 \times 10^{-5}$ & $2.98 \times 10^{-8}$ \\
40 & $5.34 \times 10^{-5}$ & $5.34 \times 10^{-5}$ & $3.72 \times 10^{-8}$ \\
\hline
\end{tabular}

TABLE 4.2. $q=2$,

Projection Approximation

\begin{tabular}{|l|c|c|c|}
\hline$n$ & $\left|\tilde{\lambda}-\lambda_{q, n, 1}\right|$ & $\left|\tilde{\lambda}-\lambda_{q, n, 2}\right|$ & $\left|\tilde{\lambda}-\hat{\lambda}_{q, n}\right|$ \\
\hline 10 & $5.29 \times 10^{-5}$ & $5.29 \times 10^{-5}$ & $9.79 \times 10^{-6}$ \\
20 & $3.81 \times 10^{-5}$ & $3.81 \times 10^{-5}$ & $5.74 \times 10^{-7}$ \\
30 & $2.00 \times 10^{-5}$ & $2.00 \times 10^{-5}$ & $7.45 \times 10^{-8}$ \\
40 & $3.37 \times 10^{-5}$ & $3.35 \times 10^{-5}$ & $8.19 \times 10^{-8}$ \\
\hline
\end{tabular}

TABLE 4.4. $q=3$,

Projection Approximation

\begin{tabular}{|l|c|c|c|}
\hline$n$ & $\left|\tilde{\lambda}-\lambda_{q, n, 1}\right|$ & $\left|\tilde{\lambda}-\lambda_{q, n, 2}\right|$ & $\left|\tilde{\lambda}-\hat{\lambda}_{q, n}\right|$ \\
\hline 10 & $1.88 \times 10^{-5}$ & $1.88 \times 10^{-5}$ & $2.98 \times 10^{-8}$ \\
20 & $6.91 \times 10^{-5}$ & $6.89 \times 10^{-5}$ & $7.45 \times 10^{-8}$ \\
30 & $5.75 \times 10^{-5}$ & $5.75 \times 10^{-5}$ & $5.96 \times 10^{-8}$ \\
40 & $1 . .99 \times 10^{-5}$ & $1.98 \times 10^{-5}$ & $5.96 \times 10^{-8}$ \\
\hline
\end{tabular}

TABLE 4.6. $q=4$,

Projection Approximation

\begin{tabular}{|l|l|l|c|}
\hline$n$ & $\left|\tilde{\lambda}-\lambda_{q, n, 1}\right|$ & $\left|\tilde{\lambda}-\lambda_{q, n, 2}\right|$ & $\left|\tilde{\lambda}-\hat{\lambda}_{q, n}\right|$ \\
\hline 10 & $1.71 \times 10^{-5}$ & $1.71 \times 10^{-5}$ & $1.04 \times 10^{-7}$ \\
20 & $6.47 \times 10^{-5}$ & $6.45 \times 10^{-5}$ & $1.04 \times 10^{-7}$ \\
30 & $3.99 \times 10^{-5}$ & $3.99 \times 10^{-5}$ & $5.96 \times 10^{-8}$ \\
40 & $1.07 \times 10^{-5}$ & $1.07 \times 10^{-5}$ & $8.94 \times 10^{-8}$ \\
\hline
\end{tabular}

\section{Calculations in Double Precision}

TABLE 4.7. $q=5$,

Nyström Approximation

\begin{tabular}{|l|c|c|c|}
\hline$n$ & $\left|\tilde{\lambda}-\lambda_{q, n, 1}\right|$ & $\left|\tilde{\lambda}-\lambda_{q, n, 2}\right|$ & $\left|\tilde{\lambda}-\hat{\lambda}_{q, n}\right|$ \\
\hline 10 & $1.72 \times 10^{-7}$ & $1.71 \times 10^{-7}$ & $1.72 \times 10^{-7}$ \\
20 & $4.22 \times 10^{-9}$ & $1.03 \times 10^{-9}$ & $2.62 \times 10^{-9}$ \\
30 & $2.11 \times 10^{-9}$ & $2.11 \times 10^{-9}$ & $2.30 \times 10^{-10}$ \\
40 & $3.01 \times 10^{-9}$ & $3.01 \times 10^{-9}$ & $4.11 \times 10^{-11}$ \\
\hline
\end{tabular}

TABle 4.9. $q=6$,

Nyström Approximation

\begin{tabular}{|l|c|c|c|}
\hline$n$ & $\left|\tilde{\lambda}-\lambda_{q, n, 1}\right|$ & $\left|\tilde{\lambda}-\lambda_{q, n, 2}\right|$ & $\left|\tilde{\lambda}-\hat{\lambda}_{q, n}\right|$ \\
\hline 10 & $7.39 \times 10^{-8}$ & $7.39 \times 10^{-8}$ & $7.37 \times 10^{-8}$ \\
20 & $4.64 \times 10^{-10}$ & $2.67 \times 10^{-9}$ & $1.10 \times 10^{-9}$ \\
30 & $2.25 \times 10^{-9}$ & $2.25 \times 10^{-9}$ & $9.66 \times 10^{-11}$ \\
40 & $8.74 \times 10^{-10}$ & $8.74 \times 10^{-10}$ & $1.73 \times 10^{-11}$ \\
\hline
\end{tabular}

TABLE 4.8. $q=5$,

Projection Approximation

\begin{tabular}{|l|c|c|c|}
\hline$n$ & $\left|\tilde{\lambda}-\lambda_{q, n, 1}\right|$ & $\left|\tilde{\lambda}-\lambda_{q, n, 2}\right|$ & $\left|\tilde{\lambda}-\hat{\lambda}_{q, n}\right|$ \\
\hline 10 & $2.57 \times 10^{-9}$ & $2.57 \times 10^{-9}$ & $1.99 \times 10^{-11}$ \\
20 & $1.30 \times 10^{-9}$ & $1.30 \times 10^{-9}$ & $1.93 \times 10^{-14}$ \\
30 & $1.63 \times 10^{-9}$ & $1.63 \times 10^{-9}$ & $2.22 \times 10^{-16}$ \\
40 & $2.24 \times 10^{-9}$ & $2.24 \times 10^{-9}$ & $5.97 \times 10^{-16}$ \\
\hline
\end{tabular}

TABle 4.10. $q=6$,

Projection Approximation

\begin{tabular}{|l|c|c|c|}
\hline$n$ & $\left|\tilde{\lambda}-\lambda_{q, n, 1}\right|$ & $\left|\tilde{\lambda}-\lambda_{q, n, 2}\right|$ & $\left|\tilde{\lambda}-\hat{\lambda}_{q, n}\right|$ \\
\hline 10 & $7.80 \times 10^{-10}$ & $7.81 \times 10^{-10}$ & $2.61 \times 10^{-13}$ \\
20 & $1.29 \times 10^{-9}$ & $1.29 \times 10^{-9}$ & $5.69 \times 10^{-16}$ \\
30 & $1.69 \times 10^{-9}$ & $1.69 \times 10^{-9}$ & $5.27 \times 10^{-16}$ \\
40 & $1.75 \times 10^{-9}$ & $1.75 \times 10^{-9}$ & $5.83 \times 10^{-16}$ \\
\hline
\end{tabular}




\section{REFERENCES}

1. K. ATKINSON, Convergence rates for approximate eigenvalues of compact operators, SIAM J. Numer. Anal., 12(1975), pp 213 - 222. MR 55:11653

2. J. H. BRAMBLE AND J. E. OSBORN, Rate of convergence estimates of nonselfadjoint eigenvalue problem, Math. Comp., 27(1973), pp 525 - 549. MR 51:2280

3. F. CHATELIN, Spectral Approximation of Linear Operators, Academic Press, New York (1983). MR 86d:65071

4. C. DE BOOR AND B. SWARTZ, Collocation approximation to eigenvalues of an ordinary differential equation: The principle of the thing, Math. Comp., 35(1980), pp. $679-694$. MR 81k:65097

5. D. DELLWO AND M.B. FRIEDMAN, Accelerated spectral analysis of compact operators, SIAM J. Numer. Anal., 21(1984), pp 1115 - 1131. MR 86b:65059

6. J. DESCLOUX, N. NASSIF AND J. RAPPAZ, On spectral approximation. Part 1. The problem of convergence, R.A.I.R.O. Numer. Anal., 12(1978), pp. 97 - 112. MR 58:3404a

7. J. DESCLOUX, N. NASSIF AND J. RAPPAZ, On spectral approximation. Part 2. Error estimates for the Galerkin method, R.A.I.R.O. Numer. Anal., 12(1978), pp. 113 - 119. MR 58:3404b

8. T. KATO, Perturbation theory for nullity, deficiency and other quantities of linear operators, J. Analyse Math., 6(1958), pp. 261 - 232. MR 21:6541

9. M. T. NAIR, On strongly stable approximations, J. Austral. Math. Soc. (Series A), 52(1992), pp. 251 - 260. MR 92k:47034

10. J. E. OSBORN, Spectral approximation for compact operators, Math. Comp., 29(1975), pp 712-725. MR 81f:65041

11. G. VAINIKKO, Uber die Konvergenz und Divergenz von Näherungsmethoden bei Eigenwertproblemen, Math. Nachr., 78(1977), pp. 145 - 164. MR 58:19122a

Department of Mathematics, Indian Institute of Technology Bombay, India

Current address: Department of Mathematics, Indian Institute of Technology Guwahati, India

E-mail address: rafik@iitg.ernet.in

E-mail address: rpk@math.iitb.ernet.in

E-mail address: bvl@math.iitb.ernet.in 